\title{
含苯并香豆素的喹唑啉-4-酮衍生物的合成及其抗肿瘤、抗菌活性
}

\author{
冯钰欣谭官海周利凯王淑霞陈华* 李小六*
}

(河北大学化学与环境科学学院 河北省化学生物学重点实验室 保定 071002))

\begin{abstract}
摘要 利用 Heck 偶联反应制备了 3,4-苯并香豆素醛，继而与邻氨基苯甲酰胺反应，设计合成了连有苯并香豆素并含氨 基侧链的哇唑啉-4-酮衍生物 $3 \mathrm{a} \sim 3 \mathrm{e}$ 和 $4 \mathrm{a}$, 并评价了化合物的抗肿瘤细胞增殖活性及抑菌活性. 化合物 $3 \mathrm{e}$ 和 $4 \mathrm{a}$ 具有中 等的抗宫颈癌和乳腺癌细胞增殖活性, $\mathrm{IC}_{50}$ 值分别为 22.63 和 $23.35 \mu \mathrm{mol} / \mathrm{L}$. 部分化合物 $(50 \mu \mathrm{g} / \mathrm{mL})$ 对大肠杆菌具有显著 的抑制活性，抑菌率在 89\%以上. 2-苯基-4-[2-(哌啶-1-基)乙氧基]喹唑啉(1b)对尖孢镰刀菌和立枯丝核菌以及 3d 对大丽 轮枝菌真菌的抑制率均为 $100 \%$.
\end{abstract}

关键词＼cjkstart喹唑啉-4-酮; 3,4-苯并香豆素; Heck 偶联; 抗肿瘤活性; 抗菌活性

\section{Synthesis of Novel Quinazolin-4-one Derivatives Bearing Benzo[c]- chromen-6-one and Their Anti-tumor and Antimicrobial Activities}

\author{
Feng, Yuxin Tan, Guanhai Zhou, Likai Wang, Shuxia Chen, Hua* Li, Xiaoliu* \\ (Key Laboratory of Chemical Biology of Hebei Province, College of Chemistry and Environmental Science, \\ Hebei University, Baoding 071002)
}

\begin{abstract}
A series of novel quinazolin-4(3H)-one derivatives 3a $\sim \mathbf{3 e}$ and $\mathbf{4 a}$ possessing 3,4-benzo[c]coumarin and amino side chain were designed and synthesized by the condensation reaction of 2-aminobenzamide and 3,4-benzo[c] coumarin aldehyde prepared by Heck coupling reaction, followed by $\mathrm{S}_{\mathrm{N}} 2$ substitution reaction with haloalkane. The compounds were evaluated for their anti proliferation activities against four tumor cells and antimicrobial activities. The results showed that $\mathbf{3 e}$ showed moderate anti Hela activity with the $\mathrm{IC}_{50}$ value of 22.63 and $23.35 \mu \mathrm{mol} / \mathrm{L}$ for $4 \mathbf{a}$ against MCF-7. Most tested compounds exhibited significant anti Escherichia coli activities, and the inhibition rate was above $89 \%$ at the concentration of 50 $\mu \mathrm{g} / \mathrm{mL}$. The inhibition rates of 2-phenyl-4-(2-(piperidin-1-yl)ethoxy)quinazoline (1b) against Fusarium oxysporum and Rhizoctonia solani and $\mathbf{3 d}$ against Verticillium dahliae are 100\%.
\end{abstract}

Keywords quinazolin-4-one; 3,4-benzo[c]coumarin; Heck coupling reaction; anti-tumor activity; antimicrobial activity

喹唑啉-4-酮是一类广谱的药效基团，其衍生物多 具有抗菌、抗炎、抗疮疾、抗结核、抗肿瘤和抗病毒等 作用 ${ }^{[1 \sim 3]}$. 其中 C-2 和 N-3 取代基的结构各异, 赋予了喹 唑啉-4-酮衍生物多样的生物活性 ${ }^{[4 \sim 7]}$. 之前, 我们按照 药效基团拼合的原理, 设计合成了 C-2 位分别为苯环和 香豆素环取代的含氨基侧链的喹唑啉-4-酮衍生物 1 和 2(图 1). 生物活性测试结果表明, 化合物 $\mathbf{2}$ 比 $\mathbf{1}$ 具有更 好的抗肿瘤细胞增殖活性, 提示 C-2 位香豆素(较大的 $\pi$ 平面芳环)取代将有利于提高此类化合物的抗肿瘤活性. 在此基础上, 我们设计了连有苯并香豆素并含氨基侧链 的喹唑啉-4-酮衍生物 3 和 4 (图 1), 尝试在 C-2 位引入共
轭平面更大的 3,4-苯并香豆素. 因此，本文采用邻氨基 苯甲酰胺和 3,4-苯并香豆素醛为原料构建喹唑啉-4-酮, 并酰胺烷基化合成目标化合物. 初步评价了该系列化合 物的抗肿瘤细胞增殖活性, 并进行了化合物 $1 \sim 3$ 的抗 菌活性测试.

\section{1 结果与讨论}

\section{$1.13,4$-苯并香豆素醛的合成}

\subsection{1 路线一}

如 Scheme 1 所示，以间甲基苯酚(5)和邻碘苯甲酸

*Corresponding authors. E-mail: lix1@hbu.cn; hua-todd@163.com

Received August 25, 2016; revised September 13, 2016; published online October 11, 2016.

Project supported by the National Natural Science Foundation of China (No. 21372060), the Natural Science Fund for Distinguished Young Scholars (Incubation) of Hebei Province (No. B2015201005).

国家自然科学基金(No. 21372060)、河北省自然科学杰出青年基金(培育) (No. 2015201005)资助项目 


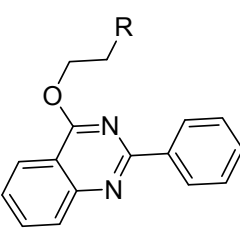

1

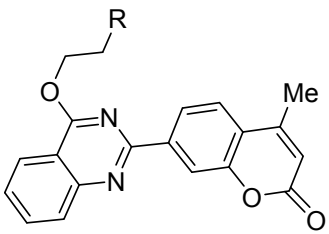

2
Known compounds<smiles>[R]CCn1c(-c2cccc3oc(=O)c4ccccc4c23)nc2ccccc2c1=O</smiles><smiles></smiles>

$3 b^{\prime} \sim 3 e^{\prime}$<smiles>[R]CCOc1nc(-c2ccc3c(c2)oc(=O)c2ccccc23)nc2ccccc12</smiles>

$4 a$

Newly designed compounds

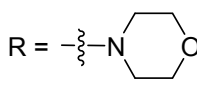

a

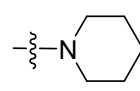

b

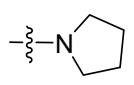

C

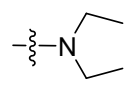

d

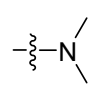

图 1 化合物 1 4 的结构

Figure 1 Structures of compounds $1 \sim 4$

(6)为起始原料, 在二环己基碳二亚胺(DCC)作用下缩合 成酯 7. 强碱条件下, 利用 Heck 分子内偶联 ${ }^{[8 \sim 10]}$ 得到难 以分离、近于等物质的量的两个 3,4-苯并香豆素同分异 构体产物 8 和 9 . 简单探讨了反应时间、温度和催化剂 用量等条件对此反应的影响(表 1). 最佳反应条件确定 为醋酸钯 0.1 equiv., 三苯基膦 0.2 equiv., 醋酸钠 2.0 equiv., $130{ }^{\circ} \mathrm{C}$ 反应 $20 \mathrm{~h}, \mathbf{8}$ 和 9 的总产率为 $60.8 \%$.

对 8 和 9 的混合物在溴代丁二酰亚胺 $(\mathrm{NBS})$ 和偶氮 二异丁腈 $(A I B N)$ 作用下溴化, 以期获得易于水解成醛 的 5 位和 7 位甲基单溴代的产物(Scheme 1) ${ }^{[11]}$. 但溴化 反应不完全, 未反应的原料全部是 $\mathbf{9}$, 增加 NBS 用量反 应也不再进行. 最终分离得到了 7-甲基双溴代化合物

表 1 Heck 反应合成 3,4-苯并香豆素 8 和 $9^{a}$

Table 1 Synthesis of 3,4-benzocoumarins 8 and 9 by Heck reaction

\begin{tabular}{cccccc}
\hline Entry & $\mathrm{Pd}(\mathrm{OAc})_{2} /$ equiv. & $\mathrm{PPh}_{3} /$ equiv. & 温度 $/{ }^{\circ} \mathrm{C}$ & $t / \mathrm{h}$ & 产率 ${ }^{b} \%$ \\
\hline 1 & 0.10 & 0.2 & 80 & 36 & 24.8 \\
2 & 0.10 & 0.2 & 100 & 36 & 55.9 \\
3 & 0.10 & 0.2 & 130 & 24 & $\mathbf{6 0 . 8}$ \\
4 & 0.10 & 0.2 & 150 & 24 & 59.4 \\
5 & 0.05 & 0.1 & 130 & 24 & 54 \\
6 & 0.05 & 0.1 & 130 & 20 & 55.7 \\
\hline$a$ & 原料 7 为 1.5 mmol, 醋酸钠为 2.0 equiv.; ${ }^{b} \mathbf{8}$ 和 $\mathbf{9}$ 的总收率.
\end{tabular}

10(主要产物)以及 $10 \%$ 的 5-甲基单溴代化合物 12 (次要 产物). 提示香豆素 7-甲基极易溴代，而 5-甲基不易溴 代. 化合物 10 的结构得到核磁共振和质谱的确证. 在化 合物 10 的 ${ }^{1} \mathrm{H}$ NMR 中, 化学位移 $\delta 7.58$ 处为单峰, $\delta 6.72$ 处积分为一个氢，确定为甲基双溴取代的 $\mathrm{CH}$. 质谱 $m / z$ : 为 $389.1[\mathrm{M}+\mathrm{Na}]^{+}$.

5-溴甲基香豆素(12)在六亚甲基四胺作用下 ${ }^{[11]}$ ，水 解得 5-醛基-3,4-苯并香豆素(13). 而 7 位的偕二溴甲基 香豆素 $(10)$ 在同等条件以及碳酸钾、氢氧化钾等条件下 均不能水解得到 7-醛基 3,4-苯并香豆素(11). 具体的反 应条件有待进一步探讨.

\subsection{2 路线二}

由于路线一无法得到 7-醛基-3,4-苯并香豆素(11), 所以我们尝试在结构中先引入醛基，以间羟基苯甲醛 14 为原料按照路线二合成 3,4-苯并香豆素醛.

如 Scheme 2 所示, 以间羟基苯甲醛(14)和邻碘苯甲 酸(6)为原料, 合成得到的酯用乙二醇保护醛基得到化 合物 15. 经 Heck 偶联反应，分离得到化合物 16 和 17. 化合物 17 在 $30 \%$ 醋酸中 $90{ }^{\circ} \mathrm{C}$ 反应 $3 \mathrm{~h}$, 合成得到 5-醛 基-3,4-苯并香豆素(13). 但同样条件下，化合物 16 却难 以脱保护得到相应的产物 11. 尝试了不同的酸性条件 脱保护，如浓度更高的醋酸溶液、盐酸、对甲苯磺酸等，

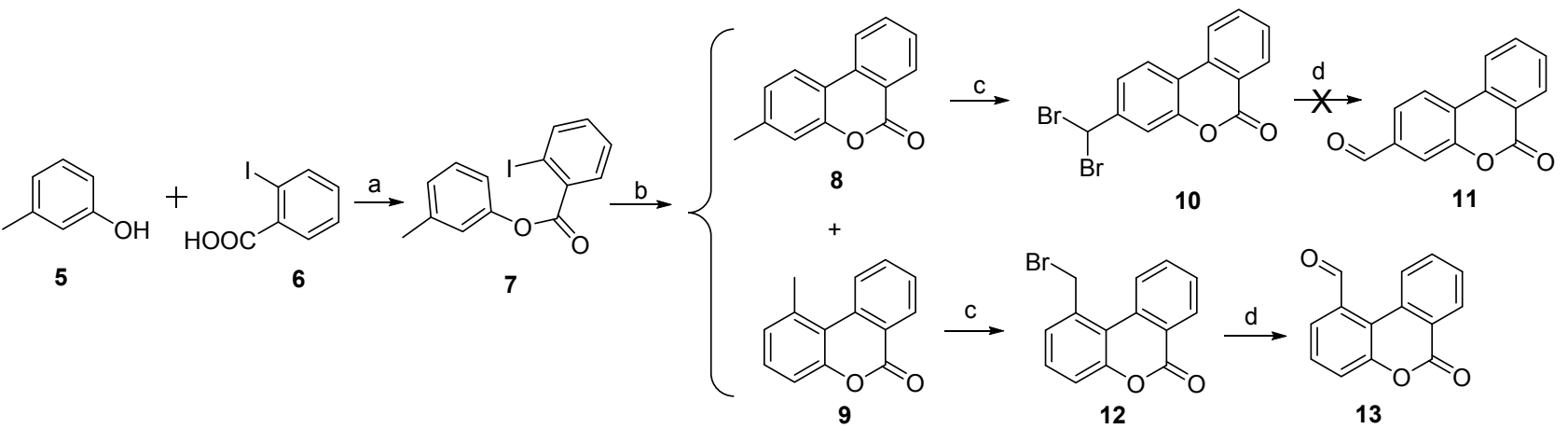

Reagents and conditions: (a) DCC, DMAP, THF, r.t., $30 \mathrm{~min}$; (b) Pd(OAc) $2 / \mathrm{PPh}_{3}, \mathrm{NaOAc}, \mathrm{DMF}, 130{ }^{\circ} \mathrm{C}, 20$ h; (c) NBS/AIBN, CCl ${ }_{4}, \mathrm{reflux}, 3 \mathrm{~h}$; (d) hexamethylenetetramine, $\mathrm{HOAc} / \mathrm{H}_{2} \mathrm{O}$, reflux, $3 \mathrm{~h}$

图式 13 ,4-苯并香豆素醛的合成(路线 1)

Scheme 1 Synthesis of the 3,4-benzo[c]coumarin aldehydes (route 1) 
均没有得到目标化合物 7-醛基-3,4-苯并香豆素(11). 其 脱保护产物主要为不溶于有机溶剂、无机酸、碱的疑是 高分子酚醛聚合物.
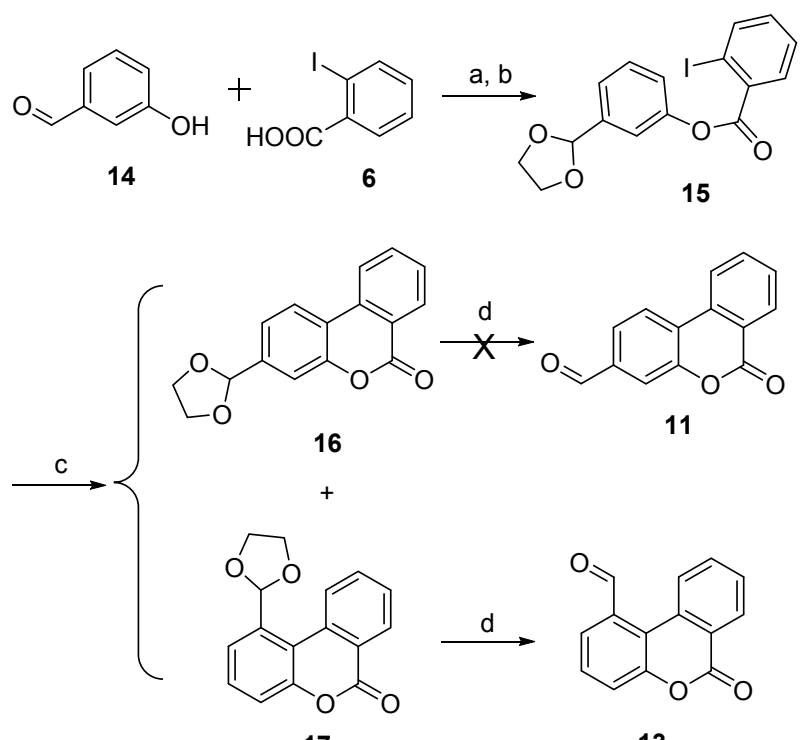

17

13

Reagents and conditions: (a) DCC, DMAP, THF, r.t., $30 \mathrm{~min}$; (b) $\mathrm{HOCH}_{2} \mathrm{CH}_{2} \mathrm{OH}$, TsOH, toluene, reflux, 4 h; (c) $\mathrm{Pd}(\mathrm{OAc})_{2} / \mathrm{PPh}_{3}$, $\mathrm{NaOAc}, \mathrm{DMF}, 130{ }^{\circ} \mathrm{C}, 20 \mathrm{~h}$; (d) $30 \% \mathrm{HOAc}$, reflux, $30 \mathrm{~min}$

图式 2 3,4-苯并香豆素醛的合成(路线 2)

Scheme 2 Synthesis of 3,4-benzo[c]coumarin aldehydes (route 2)

\section{2 化合物 $3 a \sim 3 e$ 和 $3 b^{\prime} \sim 3 e^{\prime}$ 的合成}

以邻氨基苯甲酰胺(18)和 5-醛基-3,4-苯并香豆素 (13)为原料, 在二甲基亚砜(DMSO)中 ${ }^{[12,13]}$, 一步简便合 成了连有 3,4-苯并香豆素的喹唑啉-4-酮中间体 19, 再与 卤代氨基乙烷发生 $\mathrm{S}_{\mathrm{N}} 2$ 亲核取代(烷基化)反应合成目标 产物 3 (Scheme 3).

19 的烷基化反应中, 同时得到了酰胺 $N-$-(3 位)和 $O$-(4-羰基烯醇化)烷基化产物 $3 \mathbf{a} \sim 3 \mathbf{e}$ 和 $3 \mathbf{b}^{\prime} \sim 3 \mathbf{e}^{\prime}$, 但受 到 $\mathrm{C}-2$ 取代基的位阻影响 ${ }^{[14,15]}$, 以 $N$-烷基化产物为主, 且 $N-/ O$-烷基化产物比例相差较大(表 2). 其中吗啉基取 代的 $O$-烷基化产物 3a'因产率太低, 难以分离.

表 2 化合物 19 的烷基化

Table 2 Alkylation of compound 19

\begin{tabular}{|c|c|c|c|c|}
\hline \multirow{2}{*}{ 产物 } & \multirow{2}{*}{ 总产率/\% } & \multicolumn{2}{|c|}{ 产率/\% } & \multirow{2}{*}{$N_{-}: O$} \\
\hline & & $3 a \sim 3 e$ & $3 \mathbf{b}^{\prime} \sim 3 \mathrm{e}^{\prime}$ & \\
\hline $3 \mathbf{a}$ & 55.2 & 55.2 & $-^{a}$ & $>10: 1$ \\
\hline $\mathbf{3 b} / \mathbf{3} \mathbf{b}^{\prime}$ & 49.0 & 42.7 & 6.3 & $6.8^{b}: 1$ \\
\hline $3 c / 3 c^{\prime}$ & 53.2 & 44.1 & 9.1 & $4.9: 1$ \\
\hline $\mathbf{3 d} / \mathbf{3} d^{\prime}$ & 50.5 & 42.3 & 8.2 & $5.2: 1$ \\
\hline $3 e / 3 e^{\prime}$ & 45.8 & 39.9 & 5.9 & $6.8: 1$ \\
\hline
\end{tabular}

${ }^{a}$ 未分离到纯的 $O$ 烷基化产物; ${ }^{b}$ 分离比例.

$N-/ O$-烷基化产物的结构可以通过化合物的 ${ }^{1} \mathrm{H}$ $\mathrm{NMR}$ 和 ${ }^{13} \mathrm{C}$ NMR 谱图明确区分. $N$-烷基化化合物 $\mathbf{3 a} \sim$

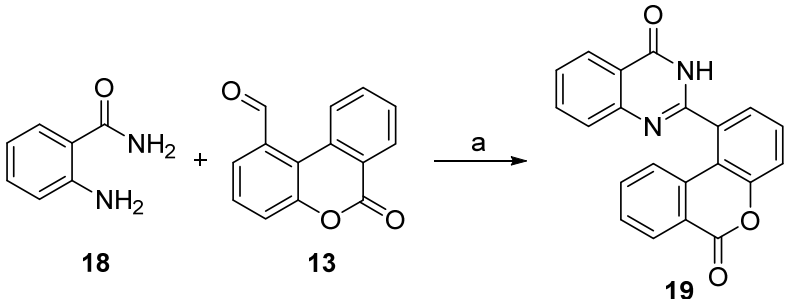<smiles>[R]CCOc1nc(-c2cccc3oc(=O)c4ccccc4c23)c2ccccc2n1</smiles>

Reagents and conditions: (a) DMSO, $120{ }^{\circ} \mathrm{C}, 4 \mathrm{~h}$; (b) acetone, $\mathrm{K}_{2} \mathrm{CO}_{3}, \mathrm{RCH}_{2} \mathrm{CH}_{2} \mathrm{Cl}, \mathrm{KI}$, reflux $16 \mathrm{~h}$

图式 3 化合物 3 的合成

Scheme 3 Synthesis of compound 3

3e 的 $\mathrm{NCH}_{2}$ 的 $\mathrm{H}$ 和 $\mathrm{C}$ 信号峰的化学位移较 $O$-烷基化化 合物 $\mathbf{3} \mathbf{b}^{\mathbf{2}} \sim \mathbf{3} \mathbf{e}^{\mathbf{\prime}}$ 的 $\mathrm{OCH}_{2}$ 的相应信号处于更高场, 且 $\mathrm{NCH}_{2}$ 在 ${ }^{1} \mathrm{H}$ NMR 中, 受到 3,4-苯并香豆素较强的屏蔽和去屏 蔽效应, 会裂分为两组信号峰(每组积分为一个氢), 而 $\mathrm{OCH}_{2}$ 不裂分仍为一组峰(积分为两个氢). 相关表征数 据见表 3 .

表 $3 N-, O$-烷基化产物结构表征

Table 3 Structural characterization of the $N$-, $\mathrm{O}$-alkylated products

\begin{tabular}{lcc}
\hline Compd. & $\delta_{\mathrm{H}}\left(N-\mathrm{CH}_{2} / O-\mathrm{CH}_{2}\right)$ & $\delta_{\mathrm{C}}\left(N-\mathrm{CH}_{2} / O-\mathrm{CH}_{2}\right)$ \\
\hline 3a & $4.17 \sim 4.14,3.64 \sim 3.61$ & 66.7 \\
3b/3b' & $4.19 \sim 4.14,3.62 \sim 3.58 / 4.63$ & $56.4 / 56.8$ \\
$\mathbf{3 c} / \mathbf{3} \mathbf{c}^{\prime}$ & $4.21 \sim 4.16,3.69 \sim 3.64 / 4.63$ & $54.0 / 54.7$ \\
$\mathbf{3 d} / \mathbf{3 d}$ & $4.13 \sim 4.08,3.49 \sim 3.45 / 4.51$ & $49.9 / 50.8$ \\
$\mathbf{3 e} / \mathbf{3} \mathbf{e}^{\prime}$ & $4.16 \sim 4.12,3.63 \sim 3.58 / 4.56$ & $56.7 / 57.5$ \\
\hline
\end{tabular}

\section{3 化合物 $4 a$ 的合成}

由于 7-醛基-3,4-苯并香豆素(11)难以合成, 所以改 变路线, 以邻氨基苯甲酰胺(18)和间羟基苯甲醛(14)为 原料, 先合成 C-2 苯酚基喹唑啉-4-酮化合物 20, 再经烷 基化, 酚羟基酯化, 并经 Heck 偶联合成 C-2 与 3,4-苯并 香豆素 7 位连接的喹唑啉-4-酮衍生物 $\mathbf{4 a}$ (Scheme 4).

需要指出的是，在化合物 $\mathbf{2 0}$ 的烷基化反应中，由于 酚羟基的存在, 得到了两组难以分离的同分异构体 $O$ 烷基化产物 21 和 $N$-烷基化产物 21-1 以及其对应双取代 
<smiles>CN(C=O)c1ccccc1C(N)=O</smiles><smiles>[R]CCOc1nc(-c2cccc(O)c2)nc2ccc([Hg])cc12</smiles><smiles>[R]CCn1c(-c2cccc(O)c2)nc2ccccc2c1=O</smiles>

21-1<smiles>[R]CCOc1cccc(-c2nc3ccccc3c(=O)n2CC[R])c1</smiles>

21-2<smiles>[R]CCOc1nc(-c2cccc(OC(=O)c3ccccc3I)c2)nc2cc(C([R])([R])[Y]([H])([H])[H])ccc12</smiles>

Reagents and conditions: (a) DMSO, $120{ }^{\circ} \mathrm{C}, 4 \mathrm{~h}$; (b) Acetone, $\mathrm{K}_{2} \mathrm{CO}_{3}, \mathrm{RCH}_{2} \mathrm{CH}_{2} \mathrm{Cl}, \mathrm{KI}$, reflux, $9 \mathrm{~h}$; (c) DCC, DMAP, THF, r.t, $12 \mathrm{~h}$; (d) $\mathrm{Pd}(\mathrm{OAc})_{2} / \mathrm{PPh}_{3}, \mathrm{NaOAc}, \mathrm{DMF}, 120{ }^{\circ} \mathrm{C}, 36 \mathrm{~h}$

图式 4 化合物 $\mathbf{4 a}$ 的合成

Scheme 4 Synthesis of compound $\mathbf{4 a}$

产物 21-3 和 21-2. 因为反应位点活性差异不大, 条件难 以控制, 分离提纯困难. 4 个取代产物比例不同, 以单 $O-$ 烷基化产物 21 为主. 通过控制反应时间以及原料氨基 链盐酸盐和碱 $\mathrm{K}_{2} \mathrm{CO}_{3}$ 的比例, 最终得到 $O$-烷基化产物 21, 产率为 $54.4 \%$.

在化合物 22 进行 Heck 偶联关环反应时, 得到 2-(7苯并香豆素)-喹唑啉-4-酮衍生物 $4 \mathbf{a}$ (主要产物)和痕量的 2-(5-苯并香豆素)-喹唑啉-4-酮衍生物 3a', 这可能是因 为在形成 3,4-苯并香豆素环时, 5 位上的空间位阻较 7 位 的大造成的. 两个异构体 $\mathbf{4 a}$ 和 $\mathbf{3} \mathbf{a}^{\prime}$ 的极性接近, 分离提 纯困难, 且 $3 \mathbf{a}^{\prime}$ 的量太少, 难以计算分离产率比例.

\section{4 抗肿瘤活性评价}

对所合成的化合物 $3 a \sim 3 e 、 4 a$ 和 $19 \sim 21$ 分别进行 了体外抑制人宫颈癌(hela)、乳腺癌(MCF-7)、肺癌
(A549)细胞活性测试，顺铂为阳性对照，结果见表 4. 总 体而言，所测试化合物对三种肿瘤细胞增殖具有中等偏 弱的抑制活性，均低于阳性对照顺铂的活性. 活性相对 较好的化合物有： $4 \mathrm{a}$ 抑制 Hela 细胞 $\left(\mathrm{IC}_{50}=22.63\right.$ $\mu \mathrm{mol} / \mathrm{L}) ; 3 \mathrm{e}$ 和 21-3 抑制 MCF-7 细胞( $\mathrm{IC}_{50}$ 分别为 23.35 和 $18.72 \mu \mathrm{mol} / \mathrm{L})$, 表现出一定的选择性.

表 4 化合物 8 和 9 的抗肿瘤活性

Table 4 Antitumor activities of compounds 8 and 9

\begin{tabular}{llll}
\hline \multirow{2}{*}{ Compd. } & \multicolumn{3}{c}{ 抗肿瘤活性 $\left(\mathrm{IC}_{50}, \mu \mathrm{mol} \cdot \mathrm{L}^{-1}\right)$} \\
\cline { 2 - 4 } & Hela & MCF-7 & A549 \\
\hline $\mathbf{1 9}$ & $31.32 \pm 0.09$ & $>50$ & $>50$ \\
$\mathbf{3 a}$ & $47.09 \pm 0.03$ & $>50$ & $38.15 \pm 0.01$ \\
$\mathbf{3 b}$ & $>50$ & $>50$ & $46.12 \pm 0.02$ \\
$\mathbf{3 c}$ & $>50$ & $>50$ & $>50$ \\
$\mathbf{3 d}$ & $>50$ & $>50$ & $>50$ \\
$\mathbf{3 e}$ & $>50$ & $\mathbf{2 3 . 3 5} \pm \mathbf{0 . 0 4}$ & $45.30 \pm 0.01$ \\
$\mathbf{4 a}$ & $\mathbf{2 2 . 6 3} \pm \mathbf{0 . 0 4}$ & $>50$ & $>50$ \\
$\mathbf{2 0}$ & $>50$ & $>50$ & $>50$ \\
$\mathbf{2 1}$ & $36.51 \pm 0.01$ & $39.07 \pm 0.02$ & $>50$ \\
$\mathbf{2 1 - 1}$ & $>50$ & $>50$ & $>50$ \\
$\mathbf{2 1 - 2}$ & $>50$ & $>50$ & $>50$ \\
$\mathbf{2 1 - 3}$ & $>50$ & $\mathbf{1 8 . 7 2} \pm \mathbf{0 . 0 1}$ & $>50$ \\
顺铂 & $9.91 \pm 0.01$ & $13.10 \pm 0.02$ & $17.00 \pm 0.01$ \\
\hline
\end{tabular}

连有 3,4-苯并香豆素的喹唑啉-4-酮化合物 3a～3e、 4a 和 19 没有得到预期的抗肿瘤活性，较之前测试的 $\mathbf{2 b} \sim \mathbf{2 e}$ (图 1)的抗肿瘤活性( $\mathrm{IC}_{50}$ 在 $10 \mu \mathrm{mol} / \mathrm{L}$ )降低, 表明 进一步延长 C-2 取代基芳香平面(三环)对化合物的抗肿 瘤活性提高意义不大. 同时，侧链氨基的不同对活性影 响不大. 而氨烷侧链的个数对化合物的活性影响也不 大，如 20 和 21 系列化合物.

\section{5 抗菌活性评价}

对之前合成的化合物 $1 \mathrm{a} \sim 1 \mathrm{c} 、 \mathbf{2 a} \sim \mathbf{2 e}$ )(图 1)和此次 合成的 $3 \mathrm{a} \sim 3 \mathrm{e}$ 分别进行了抑制植物病菌的活性测试. 所选细菌包括两株革兰氏阴性菌：大肠杆菌 (Escherichia coli)和痢疾杆菌(Shigella Castellani), 四株 植物病原菌(真菌): 链铬狍菌(Alternaria alternate)、尖狍 镰刀菌(Fusarium oxysporum)、立枯丝核菌(Rhizoctonia solani)和大丽轮枝菌(Verticillium dahliae). 以诺氟沙星 (Norfloxacin)和三唑酮(Triadimefon)为阳性对照, 测试 浓度为 $50 \mu \mathrm{g} / \mathrm{mL}$, 结果见表 5 .

实验结果表明，化合物对细菌的抑菌效果较好，对 Escherichia coli 具有显著的抑制活性，抑制率在 89\%以 上, 与 Norfloxacin 抗菌活性相当. 化合物对 Shigella Castellani 的抑制活性中等，个别化合物活性较高，如 3b 和 3c 的抑制率均为 94\%, 略低于阳性对照. 大部分 化合物对真菌也有较好的抑制作用，如对 Alternaria 
表 5 化合物 $\mathbf{1} \sim \mathbf{3}$ 的抗菌活性

Table 5 Antmicrobial activities of compounds $\mathbf{1} \sim \mathbf{3}$

\begin{tabular}{|c|c|c|c|c|c|c|}
\hline \multirow{3}{*}{ Compd. } & \multicolumn{6}{|c|}{ 抗菌抑制率/\% $\left(50 \mu \mathrm{g} \cdot \mathrm{mL}^{-1}\right)$} \\
\hline & \multicolumn{2}{|c|}{ Anti-bacteria } & \multicolumn{4}{|c|}{ Anti-fungi } \\
\hline & Escherichia coli & Shigella castellani & Alternaria alternate & Fusarium oxysporum & Rhizoctonia solani & Verticillium dahliae \\
\hline $1 \mathbf{a}$ & 99 & 66 & 82 & 65 & 68 & 44 \\
\hline $1 b$ & 98 & 74 & 73 & 100 & 100 & 54 \\
\hline $1 \mathrm{c}$ & 99 & 84 & 58 & 76 & 24 & 63 \\
\hline $2 \mathbf{a}$ & 99 & 83 & 35 & 57 & 51 & 33 \\
\hline $2 \mathbf{b}$ & 96 & 35 & 31 & 27 & 2 & 47 \\
\hline $2 \mathrm{c}$ & 99 & 15 & 57 & 46 & 21 & 37 \\
\hline 2d & 100 & 47 & 70 & 42 & 47 & 61 \\
\hline $2 e$ & 99 & 73 & 77 & 60 & 64 & 65 \\
\hline 3a & 89 & 55 & 82 & 80 & 42 & 81 \\
\hline $3 \mathbf{b}$ & 97 & 94 & 86 & 71 & 20 & 49 \\
\hline $3 c$ & 97 & 94 & 77 & 91 & 90 & 33 \\
\hline 3d & 91 & 66 & 62 & 61 & 54 & 100 \\
\hline $3 e$ & 90 & 32 & 84 & 78 & 26 & 54 \\
\hline Norfloxacin & 98 & 95 & & & & \\
\hline Triadimefon & & & 64 & 58 & 94 & 63 \\
\hline
\end{tabular}

alternate 和 Fusarium oxysporum 具有中等偏上的抑制活 性, 且多数高于阳性对照 Triadimefon; 而对 Rhizoctonia solani 和 Verticillium dahliae 具有中等偏弱的抑制作用. 少数化合物对不同的真菌有较强的选择性抑制活性, 如 1b 对 Fusarium oxysporum 和 Rhizoctonia solani 的抑制率 均为 $100 \%, \mathbf{3 d}$ 对 Verticillium dahliae 的抑制率为 $100 \%$. 连有 3,4-苯并香豆素的喹唑啉-4-酮化合物 3a $~ 3 \mathbf{e}$ 的抑 菌活性普遍高于化合物 $\mathbf{1}$ 和 $\mathbf{2}$, 且对细菌、真菌抑制活 性均较好, 表明此类化合物具有较为广谱的抑菌活性.

\section{2 结论}

本文利用 Heck 偶联反应合成了醛, 进而与邻氨基 苯甲酰胺反应, 合成了系列含氨基侧链 C-2 位 3,4-苯并 香豆素的喹唑啉-4-酮衍生物 $3 \mathbf{a} \sim 3 \mathbf{e}$ 和 $4 \mathbf{a}$. 个别化合物 $3 \mathbf{e}$ 和 $4 \mathbf{a}$ 分别具有中等的抗 MCF-7 和抗 Hela 细胞增殖 活性. 构效关系分析提示, 进一步延长 C-2 取代基共轭 平面(三环)对化合物的抗肿瘤活性提高意义不大. 此外, 化合物 1 3 均具有良好的抗菌活性, 且对细菌的抑制 活性较对真菌的更好.

\section{3 实验部分}

\section{1 仪器与试剂}

熔点由 $\mathrm{SGW}^{\circledR} \mathrm{X}-4$ 显微熔点仪(温度计未校正)测 定; 核磁共振谱用 BRUKER AC-P600 (600 MHz)型核磁 共振仪测定, TMS 为内标; 质谱(ESI)用 Agilent G6300 离子阱液相质谱联用仪; 元素分析用 HERAEUS (CHNO, rapid)型元素分析仪; 光密度用 BioRad 3550 型 酶标仪测定; 层析用硅胶(200３00 目)为青岛海洋化工
厂产品. 本文所用其他试剂均为分析纯, 无水试剂均按 常规方法处理, 水为二次蒸馏水.

\section{2 化合物 13 的合成}

\subsection{1 方法 1: 化合物 7 的合成}

$100 \mathrm{~mL}$ 圆底烧瓶中加入 $436 \mathrm{mg}(4.0 \mathrm{mmol})$ 间甲基 苯酚 $5,1.0 \mathrm{~g}(4.0 \mathrm{mmol})$ 邻碘苯甲酸 $6,832 \mathrm{mg}(4.0 \mathrm{mmol})$ 二环己基碳二亚胺(DCC), $98 \mathrm{mg}(0.8 \mathrm{mmol})$ 4-二甲氨基 吡啶(DMAP), $20 \mathrm{~mL}$ 无水四氢呋喃(THF), 室温搅拌, 薄层色谱(TLC)监测反应. $30 \mathrm{~min}$ 后, 反应完全, 抽滤反 应液并浓缩, 得到的粗产品经乙酸乙酯重结晶得到 1.05 g 2-碘苯甲酸 3-甲基苯酯(7), 淡黄色液体，产率 76.9\%. ${ }^{1} \mathrm{H}$ NMR $\left(600 \mathrm{MHz}, \mathrm{CDCl}_{3}\right) \delta: 8.09$ (d, $J=7.8 \mathrm{~Hz}, 1 \mathrm{H}$, Ar-H), 8.05 (dd, $J=1.8,7.8 \mathrm{~Hz}, 1 \mathrm{H}, \operatorname{Ar}-\mathrm{H}), 7.50(\mathrm{t}, 1 \mathrm{H}$, $J=7.8 \mathrm{~Hz}$, Ar-H), 7.34 (t, $J=7.8 \mathrm{~Hz}, 1 \mathrm{H}, \mathrm{Ar}-\mathrm{H}), 7.24$ (td, $J=1.8,7.8 \mathrm{~Hz}, 1 \mathrm{H}, \mathrm{Ar}-\mathrm{H}), 7.13 \sim 7.08$ (m, 3H, Ar-H), 2.43 (s, 3H, $\mathrm{CH}_{3}$ ); MS (ESI) $m / z: 339.2[\mathrm{M}+\mathrm{H}]^{+}$.

\section{2 .2 化合物 10 和 12 的合成}

$50 \mathrm{~mL}$ 圆底烧瓶中加入 $518 \mathrm{mg}(1.5 \mathrm{mmol})$ 化合物 7, $35 \mathrm{mg}(0.15 \mathrm{mmol})$ 醋酸钯, $80 \mathrm{mg}(0.3 \mathrm{mmol})$ 三苯基膦, $251 \mathrm{mg}$ (3.0 mmol)醋酸钠, $5 \mathrm{~mL}$ 新蒸 $N, N$-二甲基甲酰胺 $(\mathrm{DMF})$. 反应在 $130{ }^{\circ} \mathrm{C}$ 进行, TLC 监测至反应完全. 反 应液用 $5 \mathrm{~mL}$ 乙酸乙酯萃取, 有机相用饱和食盐水洗, 无水硫酸钠干燥. 抽滤并浓缩滤液, 粗产品经硅胶 (200３00 目)柱色谱 $[V$ (石油醚)： $V$ (乙酸乙酯 $)=25 ： 1]$ 分离, 得到 $518 \mathrm{mg}$ 难以分离的同分异构体 8 和 9 混合 物，收率 $55.9 \%$.

$50 \mathrm{~mL}$ 圆底烧瓶中加入 $195 \mathrm{mg}(0.9 \mathrm{mmol}) \mathbf{8}$ 和 9 的 混合物, 于 $10 \mathrm{~mL}$ 四氯化碳中加热回流至全部溶解. 分 
批加入 $180 \mathrm{mg}$ (1.0 mmol)溴代丁二酰亚胺(NBS)和 15 $\mathrm{mg}(0.09 \mathrm{mmol})$ 偶氮二异丁腈(AIBN), 继续回流 $3 \mathrm{~h}$, $\mathrm{TLC}$ 监测反应不再变化. 反应液冷却至室温, 用 $10 \mathrm{~mL}$ 乙酸乙酯萃取, 有机相用饱和食盐水洗, 无水硫酸钠干 燥. 抽滤并浓缩滤液, 粗产品经硅胶(200～300 目)柱色 谱 $[V$ (石油醚) $: V$ (乙酸乙酯 $)=15 ： 1]$ 分离得到 $\mathbf{1 0}$ 和 12.

3 -二溴甲基- $6 H$-苯并 $[c]$ 苯并二氢吡喃-6-酮(10): 无 色油状物, 产率 58.2\%. ${ }^{1} \mathrm{H}$ NMR $\left(600 \mathrm{MHz} \mathrm{CDCl}_{3}\right) \delta$ : $8.44(\mathrm{~d}, J=7.8 \mathrm{~Hz}, 1 \mathrm{H}, \mathrm{Ar}-\mathrm{H}), 8.15$ (d, $J=7.8 \mathrm{~Hz}, 1 \mathrm{H}$, Ar-H), 8.11 (d, $J=8.4 \mathrm{~Hz}, 1 \mathrm{H}, \operatorname{Ar}-\mathrm{H}), 7.89$ (t, $J=7.8 \mathrm{~Hz}$, $1 \mathrm{H}, \mathrm{Ar}-\mathrm{H}), 7.66(\mathrm{t}, J=7.2 \mathrm{~Hz}, 1 \mathrm{H}, \mathrm{Ar}-\mathrm{H}), 7.61 \sim 7.58(\mathrm{~m}$, 2H, Ar-H), 6.72 (s, 1H, CH); MS (ESI) m/z: $389.1[\mathrm{M}+$ $\mathrm{Na}]^{+}$.

1-溴甲基-6H-苯并 $[c]$ 苯并二氢吡喃-6-酮(12): 无色 油状物, 产率 $10.6 \%$. ${ }^{1} \mathrm{H} \mathrm{NMR}\left(600 \mathrm{MHz}, \mathrm{CDCl}_{3}\right) \delta: 8.53$ (t, $J=7.2 \mathrm{~Hz}, 2 \mathrm{H}, \mathrm{Ar}-\mathrm{H}), 7.96$ (t, $J=7.8 \mathrm{~Hz}, 1 \mathrm{H}, \mathrm{Ar}-\mathrm{H})$, 7.69 (d, $J=7.8 \mathrm{~Hz}, 1 \mathrm{H}, \mathrm{Ar}-\mathrm{H}), 7.48$ (t, $J=7.8 \mathrm{~Hz}, 1 \mathrm{H}$, Ar-H), 7.42 (d, J=7.2 Hz, 2H, Ar-H), 4.95 (s, 2H, $\mathrm{CH}_{2}$ ); MS (ESI) $m / z: 313.9[\mathrm{M}+\mathrm{Na}]^{+}$.

\section{2 .3 化合物 13 的合成}

$50 \mathrm{~mL}$ 圆底烧瓶中加入 $145 \mathrm{mg}(0.5 \mathrm{mmol})$ 化合物 $12,102 \mathrm{mg}(0.7 \mathrm{mmol})$ 六亚甲基四胺, $5 \mathrm{~mL}$ 水. 加热回流 至原料完全溶解后, 加入等体积的冰醋酸, 继续回流 4 $h$, TLC 监测反应完成. 反应液自然冷却至室温, 再置于 冰水中冷却, 析出大量白色固体. 抽滤得到粗品, 经乙酸 乙酯重结晶得到 $91 \mathrm{mg}$ 6-氧亚基- $6 H$-苯并 $[c]$ 苯并二氢吡 喃-1-甲醛(13), 白色固体，产率 81.2\%. m.p. 174 $176{ }^{\circ} \mathrm{C} ;{ }^{1} \mathrm{H}$ NMR (600 MHz, $\left.\mathrm{CDCl}_{3}\right) \delta: 10.55$ (s, $1 \mathrm{H}$, CHO), 8.53 (d, $J=7.8 \mathrm{~Hz}, 1 \mathrm{H}, \operatorname{Ar}-\mathrm{H}), 7.91$ (t, $J=7.8 \mathrm{~Hz}$, $1 \mathrm{H}, \mathrm{Ar}-\mathrm{H}), 7.79 \sim 7.74(\mathrm{~m}, 3 \mathrm{H}, \mathrm{Ar}-\mathrm{H}), 7.65 \sim 7.62(\mathrm{~m}, 2 \mathrm{H}$, Ar-H); MS (ESI) $m / z: 247.2[\mathrm{M}+\mathrm{Na}]^{+}$.

3.2 .4 方法 2: 化合物 15 的合成

$50 \mathrm{~mL}$ 圆底烧瓶中加入 $244 \mathrm{mg}(2.0 \mathrm{mmol})$ 间羟基苯 甲醛 14, $496 \mathrm{mg}(2.0 \mathrm{mmol})$ 邻碘苯甲酸 $6,412 \mathrm{mg}(2.0$ $\mathrm{mmol}) \mathrm{DCC}$ 和 $24 \mathrm{mg}(0.4 \mathrm{mmol}) \mathrm{DMAP}$. 于 $10 \mathrm{~mL}$ 无水 四氢呋喃中室温摚拌. $30 \mathrm{~min}$ 后, TLC 监测反应完全, 抽 滤并浓缩滤液, 粗产品在乙酸乙酯中重结晶得到 667 $\mathrm{mg}$ 邻碘苯甲酸酯.

$50 \mathrm{~mL}$ 圆底烧瓶中加入 $350 \mathrm{mg}(1.0 \mathrm{mmol})$ 上述合成 的酯, $308 \mathrm{mg}(5.0 \mathrm{mmol})$ 乙二醇, $37 \mathrm{mg}(0.2 \mathrm{mmol})$ 对甲 苯磺酸. 于 $10 \mathrm{~mL}$ 无水甲苯中回流分水. 反应 $4 \mathrm{~h}, \mathrm{TLC}$ 监测反应完全. 反应液冷却至室温, 用 $10 \mathrm{~mL}$ 乙酸乙酯 萃取, 有机相用无水硫酸钠干燥. 抽滤并浓缩滤液, 粗 产品经硅胶 $(200 \sim 300$ 目) 柱色谱 [ $V$ (石油醚)： $V($ 乙酸乙酯 $)=10: 1$ ]分离, 得到 $350 \mathrm{mg}$ 2-碘苯甲酸
3-(1,3-二氧戊环-2-基)苯酯(15), 淡黄色液体，产率 89.0\%. ${ }^{1} \mathrm{H}$ NMR $\left(600 \mathrm{MHz}, \mathrm{CDCl}_{3}\right) \delta: 8.07$ (d, $J=7.8 \mathrm{~Hz}$, Ar-H, 1H), 8.03 (dd, $J=1.8,7.8 \mathrm{~Hz}, 1 \mathrm{H}, 1 \mathrm{H}$, Ar-H), $7.49 \sim 7.44$ (m, 2H, Ar-H), 7.40 (brs, 2H, Ar-H), 7.28 (d, $J=7.8 \mathrm{~Hz}, 1 \mathrm{H}, \mathrm{Ar}-\mathrm{H}), 7.22$ (td, $J=1.8,7.8 \mathrm{~Hz}, 1 \mathrm{H}, \mathrm{Ar}-\mathrm{H})$, $5.88(\mathrm{~s}, 1 \mathrm{H}, \mathrm{CH}), 4.12 \sim 4.08\left(\mathrm{~m}, 2 \mathrm{H}, \mathrm{CH}_{2}\right), 4.05 \sim 4.03$ (m, 2H, $\mathrm{CH}_{2}$ ); $\mathrm{MS}(\mathrm{ESI}) \mathrm{m} / z: 397.2[\mathrm{M}+\mathrm{H}]^{+}$.

\subsection{5 化合物 16 和 17 的合成}

$50 \mathrm{~mL}$ 圆底烧瓶中加入 $1.7 \mathrm{~g}(4.3 \mathrm{mmol}) \mathbf{1 5}, 97 \mathrm{mg}$ (0.4 mmol)醋酸钯, $227 \mathrm{mg}(0.8 \mathrm{mmol})$ 三苯基膦, $513 \mathrm{mg}$ (6.5 mmol)醋酸钠. 于 $5 \mathrm{~mL}$ 新蒸 $\mathrm{DMF}$ 中 $130{ }^{\circ} \mathrm{C}$ 反应, TLC 监测反应结束. 反应液冷却至室温后, 用乙酸乙酯 $(5 \mathrm{~mL} \times 2)$ 萃取, 有机相用饱和食盐水洗涤, 无水硫酸钠 干燥. 粗品经硅胶(200 300 目)柱色谱 $[V$ (石油醚) : $V($ 乙酸乙酯 $)=5: 1$ ]分离, 得到 $313 \mathrm{mg} 16$ 和 $255 \mathrm{mg} 17$.

3-(1,3-二氧戊环-2-基)- $6 H$-苯并 $[c]$ 苯并二氢吡喃-6酮(16): 白色固体，产率 $27.0 \%$. m.p. $117 \sim 118{ }^{\circ} \mathrm{C} ;{ }^{1} \mathrm{H}$ NMR $\left(600 \mathrm{MHz}, \mathrm{CDCl}_{3}\right) \delta: 8.42(\mathrm{~d}, J=7.8 \mathrm{~Hz}, 1 \mathrm{H}, \mathrm{Ar}-\mathrm{H})$, $8.14(\mathrm{~d}, J=8.4 \mathrm{~Hz}, 1 \mathrm{H}, \mathrm{Ar}-\mathrm{H}), 8.09$ (d, $J=8.4 \mathrm{~Hz}, 1 \mathrm{H}$, Ar-H), 7.84 (t, $J=8.4 \mathrm{~Hz}, 1 \mathrm{H}, \mathrm{Ar}-\mathrm{H}), 7.60$ (t, $J=7.8 \mathrm{~Hz}$, $1 \mathrm{H}, \operatorname{Ar}-\mathrm{H}), 7.50(\mathrm{~s}, 1 \mathrm{H}, \operatorname{Ar}-\mathrm{H}), 7.46(\mathrm{~d}, J=8.4 \mathrm{~Hz}, 1 \mathrm{H}$, $\mathrm{Ar}-\mathrm{H}), 5.91(\mathrm{~s}, 1 \mathrm{H}, \mathrm{CH}), 4.16 \sim 4.14\left(\mathrm{~m}, 2 \mathrm{H}, \mathrm{CH}_{2}\right), 4.09 \sim$ 4.07 (m, 2H, $\mathrm{CH}_{2}$ ); MS (ESI) $m / z: 291.5[\mathrm{M}+\mathrm{Na}]^{+}$.

1-(1,3-二氧戊环-2-基)- $6 H$-苯并 $[c]$ 苯并二氢吡喃-6酮(17): 白色固体，产率 $22.0 \%$. m.p. $136 \sim 137{ }^{\circ} \mathrm{C} ;{ }^{1} \mathrm{H}$ NMR (600 MHz, $\left.\mathrm{CDCl}_{3}\right) \delta: 8.53(\mathrm{~d}, J=7.8 \mathrm{~Hz}, 1 \mathrm{H}, \mathrm{Ar}-\mathrm{H})$, $8.51(\mathrm{dd}, J=1.2,7.8 \mathrm{~Hz}, 1 \mathrm{H}, \mathrm{Ar}-\mathrm{H}), 7.87$ (td, $J=1.8,7.8$ $\mathrm{Hz}, 1 \mathrm{H}, \mathrm{Ar}-\mathrm{H}), 7.84$ (d, J=7.8 Hz, 1H, Ar-H), 7.65 (t, $J=$ $7.8 \mathrm{~Hz}, 1 \mathrm{H}, \mathrm{Ar}-\mathrm{H}), 7.54$ (t, $J=7.8 \mathrm{~Hz}, 1 \mathrm{H}, \mathrm{Ar}-\mathrm{H}), 7.45$ (d, $1 \mathrm{H}, J=7.8 \mathrm{~Hz}, \mathrm{Ar}-\mathrm{H}), 6.34$ (s, 1H, CH), 4.34 4.31(m, $\left.2 \mathrm{H}, \mathrm{CH}_{2}\right), 4.24 \sim 4.21\left(\mathrm{~m}, 2 \mathrm{H}, \mathrm{CH}_{2}\right)$; MS (ESI) $m / z: 291.5$ $[\mathrm{M}+\mathrm{Na}]^{+}$.

\section{2 .6 化合物 13 的合成}

$50 \mathrm{~mL}$ 圆底烧瓶中加入 $270 \mathrm{mg}(1.0 \mathrm{mmol}) \mathbf{1 7}, 10$

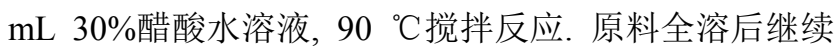
反应 $30 \mathrm{~min}$. 反应液置于冰水中冷却, 抽滤所得粗产品 用乙酸乙酯重结晶得到 $180 \mathrm{mg} \mathrm{13}$, 收率 $80 \%$.

\section{3 化合物 19 的合成}

$10 \mathrm{~mL}$ 圆底烧瓶中加入 $136 \mathrm{mg}(1.0 \mathrm{mmol})$ 邻氨基苯 甲酰胺(18)和 $269 \mathrm{mg}$ (1.2 mmol) 5-苯并香豆素醛(13), 用 $5 \mathrm{~mL}$ 二甲基亚砜(DMSO)溶解, 在 $120{ }^{\circ} \mathrm{C}$ 油浴条件 下加热搅拌, TLC 监测至反应完全. 反应液冷却至室温, 然后倒入 $100 \mathrm{~mL}$ 水中, 搅拌均匀静置, 析出白色沉淀. 粗品经重结晶 $[V$ (石油醚) $: V($ 乙酸乙酯 $)=1 ： 1]$ 得到 $369 \mathrm{mg}$ 2-(6-氧亚基- $6 H$-苯并 $[c]$ 苯并二氢吡喃-1-基)喹唑 
啉-4(3H)-酮(19), 白色固体, 产率 73.2\%. m.p. 272.1 $273.7{ }^{\circ} \mathrm{C} ;{ }^{1} \mathrm{H}$ NMR (600 MHz, DMSO- $\left.d_{6}\right) \delta: 12.68(\mathrm{~s}, 1 \mathrm{H}$, $\mathrm{NH}), 8.33$ (d, $J=7.2 \mathrm{~Hz}, 1 \mathrm{H}, \operatorname{Ar}-\mathrm{H}), 8.23(\mathrm{~d}, J=7.2 \mathrm{~Hz}$, 1H, Ar-H), 7.89 (t, $J=7.2 \mathrm{~Hz}, 1 \mathrm{H}, \mathrm{Ar}-\mathrm{H}), 7.75$ (d, $J=7.8$ $\mathrm{Hz}, 1 \mathrm{H}, \mathrm{Ar}-\mathrm{H}), 7.70$ (d, $J=7.8 \mathrm{~Hz}, 2 \mathrm{H}, \mathrm{Ar}-\mathrm{H}), 7.61 \sim 7.66$ (m, 3H, Ar-H), 7.57 (d, $J=6.6 \mathrm{~Hz}, 1 \mathrm{H}, \mathrm{Ar}-\mathrm{H}), 7.32$ (d, $J=$ $7.8 \mathrm{~Hz}, 1 \mathrm{H}, \mathrm{Ar}-\mathrm{H}) ;{ }^{13} \mathrm{C}$ NMR (150 MHz, DMSO- $\left.d_{6}\right) \delta$ : $161.6,159.9,154.3,151.0,148.7,135.0,134.7,133.2$, $132.2,130.1,130.1,129.5,127.6,127.4,127.2,126.0$, 125.0, 121.6, 121.4, 119.2, 116.2; MS (ESI) $\mathrm{m} / \mathrm{z}: 339.2$ $[\mathrm{M}-\mathrm{H}]^{+}$.

\section{4 化合物 $3 a \sim 3 e$ 和 $3 b^{\prime} \sim 3 e^{\prime}$ 的合成}

以化合物 $\mathbf{3 c}$ 和 $3 \mathbf{c}^{\prime}$ 的合成为例: $50 \mathrm{~mL}$ 圆底烧瓶中加 入 $60 \mathrm{mg}(0.3 \mathrm{mmol})$ 化合物 $\mathbf{1 9}, 80 \mathrm{mg}(0.9 \mathrm{mmol})$ 无水碳 酸钾, 再加入 $10 \mathrm{~mL}$ 新处理的丙酮, 搅拌回流 $30 \mathrm{~min}$ 后 加入 $66 \mathrm{mg}(0.6 \mathrm{mmol}) 2$-氯-乙基四氢吡咯烷盐酸盐和 催化量碘化钾, 继续回流搅拌 $16 \mathrm{~h}$, TLC 监测反应完全. 反应液冷却至室温, 抽滤除去碳酸钾, 浓缩滤液, 残留 物加 $20 \mathrm{~mL}$ 二氯甲烷(DCM) 溶解, 并用 $10 \mathrm{~mL}$ 水洗 2 次, 有机相在无水硫酸镁中干燥. 抽滤并浓缩滤液, 粗产品 经硅胶 (200 300 目)柱色谱 $[$ (二氯甲烷) $: V$ (甲醇 $)=$ $30: 1$ ]分离, 得到 $34 \mathrm{mg} \mathrm{N}$-烷基化产物 $3 \mathbf{c}$ 和 7-mg O-烷 基化产物 3c'.

以同样的方法可以得到 $3 a 、 3 b 、 3 b^{\prime} 、 3 d 、 3 d ' 、 3 e$ 和 $3 \mathrm{e}^{\mathrm{\prime}}$.

3-(2-吗啉乙基)-2-(6-氧亚基- $6 H$-苯并 $[c]$ 苯并二氢吡 喃-1-基)喹唑啉-4(3H)-酮(3a): 白色固体, 产率 55.2\%. m.p. $164.3 \sim 165.3{ }^{\circ} \mathrm{C} ;{ }^{1} \mathrm{H}$ NMR $\left(600 \mathrm{MHz}, \mathrm{CDCl}_{3}\right) \delta$ : $8.46(\mathrm{~d}, J=7.2 \mathrm{~Hz}, 1 \mathrm{H}, \mathrm{Ar}-\mathrm{H}), 8.40(\mathrm{~d}, J=7.8 \mathrm{~Hz}, 1 \mathrm{H}$, Ar-H), 7.84 (t, $J=7.2 \mathrm{~Hz}, 1 \mathrm{H}, \mathrm{Ar}-\mathrm{H}), 7.74(\mathrm{~d}, J=7.8 \mathrm{~Hz}$, 1H, Ar-H), $7.65 \sim 7.60$ (m, 2H, Ar-H), 7.57 (d, $J=7.8 \mathrm{~Hz}$, 1H, Ar-H), 7.55 (t, $J=7.8 \mathrm{~Hz}, 1 \mathrm{H}, \mathrm{Ar}-\mathrm{H}), 7.48$ (t, $J=7.2$ $\mathrm{Hz}, 1 \mathrm{H}, \mathrm{Ar}-\mathrm{H}), 7.41$ (d, $J=6.6 \mathrm{~Hz}, 1 \mathrm{H}, \mathrm{Ar}-\mathrm{H}), 7.18$ (d, $J=$ $8.4 \mathrm{~Hz}, 1 \mathrm{H}, \mathrm{Ar}-\mathrm{H}), 4.17 \sim 4.14\left(\mathrm{~m}, 1 \mathrm{H}, \mathrm{CH}_{2}\right), 3.64 \sim 3.61$ (m, $1 \mathrm{H}, \mathrm{CH}_{2}$ ), 3.46 (brs, $\left.4 \mathrm{H}, \mathrm{CH}_{2}\right), 2.44 \sim 2.40(\mathrm{~m}, 2 \mathrm{H}$, $\mathrm{CH}_{2}$ ), 2.17 (brs, $2 \mathrm{H}, \mathrm{CH}_{2}$ ), 2.12 (brs, $2 \mathrm{H}, \mathrm{CH}_{2}$ ); ${ }^{13} \mathrm{C} \mathrm{NMR}$ $\left(150 \mathrm{MHz}, \mathrm{CDCl}_{3}\right) \delta: 162.0,160.3,155.3,151.9,147.3$, $135.2,135.0,133.3,132.1,131.3,130.2,129.7,128.0$, $127.9,127.1,124.3,122.3,121.1,120.1,116.6,66.7,56.3$, 53.6, 42.7; MS (ESI) $m / z: 476.0[\mathrm{M}+\mathrm{Na}]^{+}$. Anal. calcd for $\mathrm{C}_{27} \mathrm{H}_{23} \mathrm{~N}_{3} \mathrm{O}_{4}$ : C 71.51, H 5.11, N 9.27; found C 71.60, H $5.23, \mathrm{~N} 9.34$.

3-[2-(哌啶-1-基)乙基]-2-(6-氧亚基- $6 H$-苯并 $[c$ 苯并 二氢吡喃-1-基)喹唑啉-4(3H)-酮(3b): 白色固体, 产率 42.7\%. m.p. $159.7 \sim 161.1{ }^{\circ} \mathrm{C} ;{ }^{1} \mathrm{H}$ NMR $(600 \mathrm{MHz}$,
$\left.\mathrm{CDCl}_{3}\right) \delta: 8.46(\mathrm{~d}, J=7.8 \mathrm{~Hz}, 1 \mathrm{H}, \mathrm{Ar}-\mathrm{H}), 8.40(\mathrm{~d}, J=7.8$ $\mathrm{Hz}, 1 \mathrm{H}, \mathrm{Ar}-\mathrm{H}), 7.82$ (t, $J=7.2 \mathrm{~Hz}, 1 \mathrm{H}, \mathrm{Ar}-\mathrm{H}), 7.73$ (d, $J=$ $8.4 \mathrm{~Hz}, 1 \mathrm{H}, \mathrm{Ar}-\mathrm{H}), 7.64 \sim 7.59$ (m, 2H, Ar-H), 7.56 (d, $J=$ $8.4 \mathrm{~Hz}, 1 \mathrm{H}, \mathrm{Ar}-\mathrm{H}), 7.54$ (t, $J=7.8 \mathrm{~Hz}, 1 \mathrm{H}, \mathrm{Ar}-\mathrm{H}), 7.47$ (t, $J=7.2 \mathrm{~Hz}, 1 \mathrm{H}, \mathrm{Ar}-\mathrm{H}), 7.40$ (d, $J=7.2 \mathrm{~Hz}, 1 \mathrm{H}, \mathrm{Ar}-\mathrm{H}), 7.17$ (d, $J=8.4 \mathrm{~Hz}, 1 \mathrm{H}, \operatorname{Ar}-\mathrm{H}), 4.19 \sim 4.14\left(\mathrm{~m}, 1 \mathrm{H}, \mathrm{CH}_{2}\right)$, $3.62 \sim 3.58\left(\mathrm{~m}, 1 \mathrm{H}, \mathrm{CH}_{2}\right), 2.45 \sim 2.41\left(\mathrm{~m}, 1 \mathrm{H}, \mathrm{CH}_{2}\right)$, $2.38 \sim 2.33\left(\mathrm{~m}, 1 \mathrm{H}, \mathrm{CH}_{2}\right.$ ), 2.14 (brs, $2 \mathrm{H}, \mathrm{CH}_{2}$ ), 2.07 (brs, $2 \mathrm{H}, \mathrm{CH}_{2}$ ), 1.34 (brs, 4H, $\mathrm{CH}_{2}$ ), 1.28 (brs, $2 \mathrm{H}, \mathrm{CH}_{2}$ ); ${ }^{13} \mathrm{C}$ NMR $\left(150 \mathrm{MHz}, \mathrm{CDCl}_{3}\right) \delta: 162.0,160.4,155.5,151.9$, $147.3,135.2,134.9,133.4,132.2,131.3,130.2,129.6$, 127.9, 127.1, 124.3, 122.2, 121.2, 120.0, 116.5, 56.4, 54.6, 43.1, 31.7, 25.7, 24.0; MS (ESI) $m / z: 492.1[\mathrm{M}+\mathrm{K}]^{+}$. Anal. calcd for $\mathrm{C}_{28} \mathrm{H}_{25} \mathrm{~N}_{3} \mathrm{O}_{3}$ : C 74.48, H 5.58, N 9.31; found $\mathrm{C} 74.29, \mathrm{H} 5.67, \mathrm{~N} 9.36$.

1-\{4-[2-(哌啶-1-基)乙氧基]喹唑啉-2-基 $\}$ - $6 H$-苯并 [c]苯并二氢吡喃-6-酮 $\left(\mathbf{B b}^{\prime}\right)$ : 白色固体, 产率 $6.3 \%$. m.p. $182.6 \sim 183.8{ }^{\circ} \mathrm{C} ;{ }^{1} \mathrm{H}$ NMR $\left(600 \mathrm{MHz}, \mathrm{CDCl}_{3}\right) \delta: 8.40(\mathrm{~d}$, $J=7.8 \mathrm{~Hz}, 1 \mathrm{H}, \mathrm{Ar}-\mathrm{H}), 8.28(\mathrm{~d}, J=8.4 \mathrm{~Hz}, 1 \mathrm{H}, \mathrm{Ar}-\mathrm{H}), 8.00$ (d, $J=8.4 \mathrm{~Hz}, 1 \mathrm{H}, \mathrm{Ar}-\mathrm{H}), 7.92(\mathrm{t}, J=7.2 \mathrm{~Hz}, 1 \mathrm{H}, \mathrm{Ar}-\mathrm{H})$, 7.67 (t, $J=7.8 \mathrm{~Hz}, 1 \mathrm{H}, \mathrm{Ar}-\mathrm{H}), 7.59 \sim 7.55$ (m, 2H, Ar-H), 7.49 (dd, $J=7.2,1.8 \mathrm{~Hz}, 1 \mathrm{H}$, Ar-H), 7.46 (t, $J=7.2 \mathrm{~Hz}$, 1H, Ar-H), 7.32 (t, $J=7.8 \mathrm{~Hz}, 1 \mathrm{H}, \mathrm{Ar}-\mathrm{H}), 6.91$ (d, $J=8.4$ $\mathrm{Hz}, 1 \mathrm{H}, \mathrm{Ar}-\mathrm{H}$ ), 4.63 (brs, $2 \mathrm{H}, \mathrm{CH}_{2}$ ), 2.83 (brs, $2 \mathrm{H}, \mathrm{CH}_{2}$ ), 2.50 (brs, $4 \mathrm{H}, \mathrm{CH}_{2}$ ), 1.64 (brs, $4 \mathrm{H}, \mathrm{CH}_{2}$ ), 1.45 (brs, $2 \mathrm{H}$, $\left.\mathrm{CH}_{2}\right) ;{ }^{13} \mathrm{C} \mathrm{NMR}\left(150 \mathrm{MHz}, \mathrm{CDCl}_{3}\right) \delta: 167.1,163.5,161.5$, $152.5,152.3,138.7,134.9,134.8,133.8,130.7,130.2$, 129.0, 128.6, 128.3, 127.1, 124.2, 122.9, 119.1, 117.2, 115.5, 56.8, 54.8, 24.8, 24.8, 23.6, 23.5; MS (ESI) $\mathrm{m} / \mathrm{z}$ : $490.2[\mathrm{M}+\mathrm{K}]^{+}$. Anal. calcd for $\mathrm{C}_{28} \mathrm{H}_{25} \mathrm{~N}_{3} \mathrm{O}_{3}: \mathrm{C} 74.48, \mathrm{H}$ 5.58, N 9.31; found C 74.36, H 5.52, N 9.25.

3-[2-(四氢吡咯-1-基)乙基]-2-(6-氧亚基-6H-苯并 [c] 苯并二氢吡喃-1-基)喹唑啉-4(3H)-酮(3c)：白色固体, 产 率 44.1\%. m.p. $77.7 \sim 78.9{ }^{\circ} \mathrm{C} ;{ }^{1} \mathrm{H}$ NMR $(600 \mathrm{MHz}$, $\left.\mathrm{CDCl}_{3}\right) \delta: 8.45$ (d, $\left.J=7.2 \mathrm{~Hz}, 1 \mathrm{H}, \mathrm{Ar}-\mathrm{H}\right), 8.39$ (t, $J=8.4$ $\mathrm{Hz}, 1 \mathrm{H}, \mathrm{Ar}-\mathrm{H}), 7.82$ (t, $J=6.6 \mathrm{~Hz}, 1 \mathrm{H}, \mathrm{Ar}-\mathrm{H}), 7.73$ (d, $J=$ $7.8 \mathrm{~Hz}, 1 \mathrm{H}, \mathrm{Ar}-\mathrm{H}), 7.64 \sim 7.59$ (m, 2H, Ar-H), 7.55 (d, $J=$ $6.6 \mathrm{~Hz}, 1 \mathrm{H}, \mathrm{Ar}-\mathrm{H}), 7.53$ (t, J=7.8 Hz, 2H, Ar-H), 7.47 (t, $J=6.6 \mathrm{~Hz}, 2 \mathrm{H}, \mathrm{Ar}-\mathrm{H}), 7.40$ (t, $J=6.6 \mathrm{~Hz}, 2 \mathrm{H}, \mathrm{Ar}-\mathrm{H}), 7.19$ (t, $J=8.4 \mathrm{~Hz}, 1 \mathrm{H}, \mathrm{Ar}-\mathrm{H}), 4.21 \sim 4.16\left(\mathrm{~m}, 1 \mathrm{H}, \mathrm{CH}_{2}\right), 3.69 \sim$ $3.64\left(\mathrm{~m}, 1 \mathrm{H}, \mathrm{CH}_{2}\right), 2.63 \sim 2.59$ (s, $\left.2 \mathrm{H}, \mathrm{CH}_{2}\right), 2.18$ (brs, $4 \mathrm{H}$, $\left.\mathrm{CH}_{2}\right), 1.52\left(\mathrm{~m}, 4 \mathrm{H}, \mathrm{CH}_{2}\right) ;{ }^{13} \mathrm{C}$ NMR $\left(150 \mathrm{MHz}, \mathrm{CDCl}_{3}\right) \delta$ : $162,0,160.4,155.5,151.9,147.3,135.0,134.9,133.4$, $132.2,131.2,130.1,129.5,127.9,127.9,127.1,127.1$, $124.4,122.2,121.2,120.0,116.6,54.0,53.6,44.6,23.4$; 
MS (ESI) $m / z: 438.2[\mathrm{M}+\mathrm{H}]^{+}$. Anal. calcd for $\mathrm{C}_{27} \mathrm{H}_{23^{-}}$ $\mathrm{N}_{3} \mathrm{O}_{3}$ : C 74.12, H 5.30, N 9.60; found C 74.09, H 5.36, N 9.71.

1-\{4-[2-(四氢吡咯-1-基)乙氧基]喹唑啉-2-基 $\}-6 H$ 苯并 $[c]$ 苯并二氢吡喃-6-酩 $\left(3 \mathbf{c}^{\prime}\right)$ : 白色固体, 产率 $9.1 \%$. m.p. 101.7 102.5 ${ }^{\circ} \mathrm{C}$; ${ }^{1} \mathrm{H}$ NMR (600 MHz, $\left.\mathrm{CDCl}_{3}\right) \delta$ : 8.40 (d, $J=7.8 \mathrm{~Hz}, 1 \mathrm{H}, \operatorname{Ar}-\mathrm{H}), 8.30$ (d, $J=8.4 \mathrm{~Hz}, 1 \mathrm{H}$, Ar-H), 8.00 (d, $J=8.4 \mathrm{~Hz}, 1 \mathrm{H}, \mathrm{Ar}-\mathrm{H}), 7.92$ (t, $J=7.8 \mathrm{~Hz}$, 1H, Ar-H), 7.67 (t, J=7.8 Hz, 1H, Ar-H), 7.59 7.55 (m, 2H, Ar-H), 7.49 (d, $J=7.2 \mathrm{~Hz}, 1 \mathrm{H}, \mathrm{Ar}-\mathrm{H}), 7.45$ (t, $J=7.8$ $\mathrm{Hz}, 1 \mathrm{H}, \mathrm{Ar}-\mathrm{H}), 7.32$ (t, $J=7.8 \mathrm{~Hz}, 1 \mathrm{H}, \mathrm{Ar}-\mathrm{H}), 6.90$ (d, $J=$ $8.4 \mathrm{~Hz}, 1 \mathrm{H}, \mathrm{Ar}-\mathrm{H}$ ), 4.63 (brs, 2H, $\mathrm{CH}_{2}$ ), 2.92 (brs, 2H, $\mathrm{CH}_{2}$ ), 2.67 (brs, 4H, $\mathrm{CH}_{2}$ ), 1.83 (brs, $4 \mathrm{H}, \mathrm{CH}_{2}$ ); ${ }^{13} \mathrm{C} \mathrm{NMR}$ $\left(150 \mathrm{MHz}, \mathrm{CDCl}_{3}\right) \delta: 163.4,161.2,152.2,152.0,138.6$, $134.6,134.4,133.5,130.4,129.8,128.6,128.3,127.9$, $127.8,126.8,124.0,122.6,118.7,117.0,115.4,54.7,54.3$, 29.8, 23.7; MS (ESI) $m / z: 476.1[\mathrm{M}+\mathrm{K}]^{+}$. Anal. calcd for $\mathrm{C}_{27} \mathrm{H}_{23} \mathrm{~N}_{3} \mathrm{O}_{3}$ : C 74.12, H 5.30, N 9.60; found $\mathrm{C} 74.16, \mathrm{H}$ $5.35, \mathrm{~N} 9.68$.

3-[2-(二乙胺基)乙基]-2-(6-氧亚基- $6 H$-苯并 $[c]$ 苯并 二氢吡喃-1-基)喹唑啉-4(3H)-酮(3d): 白色固体, 产率 42.3\%. m.p. $149.7 \sim 150.9{ }^{\circ} \mathrm{C} ;{ }^{1} \mathrm{H}$ NMR $(600 \mathrm{MHz}$, $\left.\mathrm{CDCl}_{3}\right) \delta: 8.46(\mathrm{~d}, J=7.8 \mathrm{~Hz}, 1 \mathrm{H}, \mathrm{Ar}-\mathrm{H}), 8.39$ (t, $J=6.6$ $\mathrm{Hz}, 2 \mathrm{H}, \mathrm{Ar}-\mathrm{H}), 7.83$ (t, $J=6.6 \mathrm{~Hz}, 2 \mathrm{H}, \mathrm{Ar}-\mathrm{H}), 7.74$ (t, $J=$ $6.6 \mathrm{~Hz}, 1 \mathrm{H}, \mathrm{Ar}-\mathrm{H}), 7.57 \sim 7.61(\mathrm{~m}, 2 \mathrm{H}, \mathrm{Ar}-\mathrm{H}), 7.48(\mathrm{t}, J=$ $6.6 \mathrm{~Hz}, 2 \mathrm{H}, \mathrm{Ar}-\mathrm{H}), 7.40$ (t, $J=8.4 \mathrm{~Hz}, 2 \mathrm{H}, \mathrm{Ar}-\mathrm{H}), 7.18$ (d, $J=8.4 \mathrm{~Hz}, 1 \mathrm{H}, \mathrm{Ar}-\mathrm{H}), 4.13 \sim 4.08\left(\mathrm{~m}, 1 \mathrm{H}, \mathrm{CH}_{2}\right), 3.49 \sim$ $3.45\left(\mathrm{~m}, 1 \mathrm{H}, \mathrm{CH}_{2}\right), 2.58 \sim 2.54\left(\mathrm{~m}, 1 \mathrm{H}, \mathrm{CH}_{2}\right), 2.45 \sim 2.40$ (m, 1H, $\left.\mathrm{CH}_{2}\right), 2.30 \sim 2.21\left(\mathrm{~m}, 4 \mathrm{H}, \mathrm{CH}_{2}\right), 0.75$ (brs, 6H, $\left.\mathrm{CH}_{3}\right) ;{ }^{13} \mathrm{C}$ NMR $\left(150 \mathrm{MHz}, \mathrm{CDCl}_{3}\right) \delta: 162.0,160.3,155.5$, $151.9,147.3,133.2,129.9,128.9,128.0,126.9,124.2$, $122.2,121.2,120.0,116.5,49.9,47.6,44.2,11.9$; MS (ESI) $m / z: 440.8[\mathrm{M}+\mathrm{H}]^{+}$. Anal. calcd for $\mathrm{C}_{27} \mathrm{H}_{25} \mathrm{~N}_{3} \mathrm{O}_{3}: \mathrm{C}$ 73.78, H 5.73, N 9.56; found C 73.90, H 5.81, N 9.49.

1-\{4-[2-(二乙胺基)乙氧基]喹唑啉-2-基 $\}-6 H$-苯并 [c]苯并二氢吡喃-6-酮(3d'): 白色固体, 产率 8.2\%. m.p. $162.8 \sim 164.2{ }^{\circ} \mathrm{C} ;{ }^{1} \mathrm{H}$ NMR $\left(600 \mathrm{MHz}, \mathrm{CDCl}_{3}\right) \delta: 8.39(\mathrm{~d}$, $J=7.8 \mathrm{~Hz}, 1 \mathrm{H}, \mathrm{Ar}-\mathrm{H}), 8.26$ (d, $J=8.4 \mathrm{~Hz}, 1 \mathrm{H}, \mathrm{Ar}-\mathrm{H}), 7.99$ (d, $J=8.4 \mathrm{~Hz}, 1 \mathrm{H}, \mathrm{Ar}-\mathrm{H}), 7.91(\mathrm{t}, J=7.2 \mathrm{~Hz}, 1 \mathrm{H}, \mathrm{Ar}-\mathrm{H})$, 7.66 (t, $J=7.8 \mathrm{~Hz}, 1 \mathrm{H}, \mathrm{Ar}-\mathrm{H}), 7.57$ (t, $J=7.8 \mathrm{~Hz}, 2 \mathrm{H}$, Ar-H), 7.49 (d, $J=7.2, \mathrm{~Hz}, 1 \mathrm{H}, \mathrm{Ar}-\mathrm{H}), 7.45$ (t, $J=7.8 \mathrm{~Hz}$, $1 \mathrm{H}, \mathrm{Ar}-\mathrm{H}), 7.31$ (t, $J=7.2 \mathrm{~Hz}, 1 \mathrm{H}, \mathrm{Ar}-\mathrm{H}), 6.90$ (d, $J=8.4$ $\mathrm{Hz}, 1 \mathrm{H}, \mathrm{Ar}-\mathrm{H}$ ), 4.51 (brs, 2H, $\mathrm{CH}_{2}$ ), 2.85 (brs, 2H, $\mathrm{CH}_{2}$ ), 2.59 (brs, 4H, $\mathrm{CH}_{2}$ ), 1.01 (brs, $\left.6 \mathrm{H}, \mathrm{CH}_{3}\right) ;{ }^{13} \mathrm{C}$ NMR $(150$ $\left.\mathrm{MHz}, \mathrm{CDCl}_{3}\right) \delta: 167.2,163.4,161.3,152.1,151.9,138.6$,
$134.5,133.5,130.4,129.8,128.6,128.3,127.9,127.7$, $126.8,123.9,122.5,118.6,116.9,115.3,50.8,48.0,47.7$, 11.9, 11.7; MS (ESI) $m / z: 440.3[\mathrm{M}+\mathrm{H}]^{+}$. Anal. calcd for $\mathrm{C}_{27} \mathrm{H}_{25} \mathrm{~N}_{3} \mathrm{O}_{3}$ : C 73.78, H 5.73, N 9.56; found $\mathrm{C} 73.84, \mathrm{H}$ $5.76, \mathrm{~N} 9.62$.

3-[2-(二甲胺基)乙基]-2-(6-氧亚基- $6 H$-苯并 $[c]$ 苯并 二氢吡喃-1-基)喹唑啉-4(3H)-酮(3e)：白色固体，产率 $39.9 \%$. m.p. $157.7 \sim 159.1{ }^{\circ} \mathrm{C} ;{ }^{1} \mathrm{H}$ NMR $(600 \mathrm{MHz}$, $\left.\mathrm{CDCl}_{3}\right) \delta: 8.45(\mathrm{~d}, J=7.8 \mathrm{~Hz}, 1 \mathrm{H}, \mathrm{Ar}-\mathrm{H}), 8.41(\mathrm{t}, J=8.4$ $\mathrm{Hz}, 2 \mathrm{H}, \mathrm{Ar}-\mathrm{H}), 7.83$ (d, $J=6.6 \mathrm{~Hz}, 2 \mathrm{H}, \mathrm{Ar}-\mathrm{H}), 7.74$ (d, $J=$ $7.8 \mathrm{~Hz}, 2 \mathrm{H}, \mathrm{Ar}-\mathrm{H}), 7.57$ (d, J=7.8 Hz, 2H, Ar-H), 7.48 (t, $J=6.6 \mathrm{~Hz}, 2 \mathrm{H}, \mathrm{Ar}-\mathrm{H}), 7.40$ (d, $J=6.6 \mathrm{~Hz}, 2 \mathrm{H}, \mathrm{Ar}-\mathrm{H}), 7.22$ $(\mathrm{t}, J=7.8 \mathrm{~Hz}, 1 \mathrm{H}, \mathrm{Ar}-\mathrm{H}), 4.16 \sim 4.12\left(\mathrm{~m}, 1 \mathrm{H}, \mathrm{CH}_{2}\right), 3.63 \sim$ $3.58\left(\mathrm{~m}, 1 \mathrm{H}, \mathrm{CH}_{2}\right), 2.45 \sim 2.41\left(\mathrm{~m}, 1 \mathrm{H}, \mathrm{CH}_{2}\right), 2.35 \sim 2.31$ $\left(\mathrm{m}, 1 \mathrm{H}, \mathrm{CH}_{2}\right), 1.91\left(\mathrm{~s}, 6 \mathrm{H}, \mathrm{CH}_{3}\right) ;{ }^{13} \mathrm{C} \mathrm{NMR}(150 \mathrm{MHz}$, $\left.\mathrm{CDCl}_{3}\right) \delta: 162.1,160.4,155.5,151.9,147.3,135.1,135.0$, $133.3,132.1,131.2,130.2,129.6,127.9,127.2,127.1$, $124.4,122.2,121.2,120.0,116.6,56.7,45.3,43.6$; MS (ESI) $m / z$ : $412.6[\mathrm{M}+\mathrm{H}]^{+}$. Anal. calcd for $\mathrm{C}_{25} \mathrm{H}_{21} \mathrm{~N}_{3} \mathrm{O}_{3}: \mathrm{C}$ 72.98, H 5.14, N 10.21; found C 73.05, H 5.19, N 10.32 .

1-\{4-[2-(二甲胺基)乙氧基]喹唑啉-2-基\}-6H-苯并 [c]苯并二氢吡喃-6-酩(3e'): 白色固体，产率 5.9\%. m.p. $179.3 \sim 180.2{ }^{\circ} \mathrm{C} ;{ }^{1} \mathrm{H}$ NMR $\left(600 \mathrm{MHz}, \mathrm{CDCl}_{3}\right) \delta: 8.40(\mathrm{~d}$, $J=7.2 \mathrm{~Hz}, 1 \mathrm{H}, \mathrm{Ar}-\mathrm{H}), 8.30$ (d, $J=8.4 \mathrm{~Hz}, 1 \mathrm{H}, \mathrm{Ar}-\mathrm{H}), 7.99$ (d, $J=8.4 \mathrm{~Hz}, 1 \mathrm{H}, \mathrm{Ar}-\mathrm{H}), 7.92$ (t, $J=7.2 \mathrm{~Hz}, 1 \mathrm{H}, \mathrm{Ar}-\mathrm{H})$, $7.66(\mathrm{t}, J=7.8 \mathrm{~Hz}, 1 \mathrm{H}, \mathrm{Ar}-\mathrm{H}), 7.59 \sim 7.55$ (m, 2H, Ar-H), 7.49 (dd, $J=7.2,2.4 \mathrm{~Hz}, 1 \mathrm{H}, \mathrm{Ar}-\mathrm{H}), 7.45$ (t, $J=7.8 \mathrm{~Hz}$, 1H, Ar-H), 7.32 (t, $J=7.2 \mathrm{~Hz}, 1 \mathrm{H}, \mathrm{Ar}-\mathrm{H}), 6.90$ (d, $J=8.4$ $\mathrm{Hz}, 1 \mathrm{H}, \mathrm{Ar}-\mathrm{H}$ ), 4.56 (t, $J=5.4 \mathrm{~Hz}, 2 \mathrm{H}, \mathrm{CH}_{2}$ ), 2.75 (brs, 2H, $\mathrm{CH}_{2}$ ), 2.32 (brs, $6 \mathrm{H}, \mathrm{CH}_{3}$ ); ${ }^{13} \mathrm{C} \mathrm{NMR}\left(150 \mathrm{MHz}, \mathrm{CDCl}_{3}\right) \delta$ : $167.1,163.3,161.3,152.1,151.9,138.6,134.5,134.4$, $133.5,130.4,129.8,128.6,128.2,127.9,127.8,126.8$, $124.0,122.5,118.7,117.0,115.3,57.5,45.5,43.5$; MS (ESI) $m / z$ : $450.9[\mathrm{M}+\mathrm{K}]^{+}$. Anal. calcd for $\mathrm{C}_{25} \mathrm{H}_{21} \mathrm{~N}_{3} \mathrm{O}_{3}: \mathrm{C}$ 72.98, H 5.14, N 10.21; found C 73.04, H 5.12, N 10.25.

\section{5 化合物 20 的合成}

$100 \mathrm{~mL}$ 圆底烧瓶中加入 $680 \mathrm{mg}(1.0 \mathrm{mmol})$ 邻氨基 苯甲酰胺(18), $732 \mathrm{mg}$ (1.2 mmol)间羟基苯甲醛(14), 25 $\mathrm{mL}$ DMSO 溶解, $120{ }^{\circ} \mathrm{C}$ 条件下搅拌, TLC 监测反应完 全. 反应液冷却至室温, 然后倒入 $500 \mathrm{~mL}$ 水中, 搅拌均 匀后静置, 析出白色沉淀。粗品经重结晶 $[V($ 二氯甲 烷)： $V$ (甲醇 $)=5 ： 1$ ]得到 $996 \mathrm{mg}$ 2-(3-着基苯基)喹唑 啉-4(3H)-酮(20), 白色固体, 产率 83.7\%. m.p. 239.1 $240.9{ }^{\circ} \mathrm{C} ;{ }^{1} \mathrm{H}$ NMR (600 MHz, DMSO- $\left.d_{6}\right) \delta$ : 12.45 (s, $1 \mathrm{H}$, NH), 9.79 (s, 1H, OH), 8.15 (d, J=7.8 Hz, 1H, Ar-H), 7.82 
(t, $J=7.8 \mathrm{~Hz}, 1 \mathrm{H}, \mathrm{Ar}-\mathrm{H}), 7.72(\mathrm{~d}, J=7.8 \mathrm{~Hz}, 1 \mathrm{H}, \mathrm{Ar}-\mathrm{H})$, 7.60 (d, $J=8.4 \mathrm{~Hz}, 2 \mathrm{H}$, Ar-H), $7.51(\mathrm{t}, J=7.2 \mathrm{~Hz}, 1 \mathrm{H}$, Ar-H), 7.34 (t, $J=7.8 \mathrm{~Hz}, 1 \mathrm{H}$, Ar-H), $6.98(\mathrm{~d}, J=7.2 \mathrm{~Hz}$, $1 \mathrm{H}, \mathrm{Ar}-\mathrm{H}) ;{ }^{13} \mathrm{C}$ NMR $\left(150 \mathrm{MHz}, \mathrm{DMSO}-d_{6}\right) \delta: 162.2$, 157.5, 152.4, 148.7, 134.6, 134.1, 129.7, 127.4, 126.5, $125.9,121.0,118.5,118.4,114.6$; MS (ESI) $m / z: 277.8$ $[\mathrm{M}+\mathrm{K}]^{+}$.

\section{6 化合物 21 的合成}

$250 \mathrm{~mL}$ 圆底烧瓶中加入 $2.38 \mathrm{~g}(10 \mathrm{mmol})$ 化合物 $\mathbf{2 0}$, $2.07 \mathrm{~g}(15 \mathrm{mmol})$ 无水碳酸钾. 于 $100 \mathrm{~mL}$ 新处理的丙酮

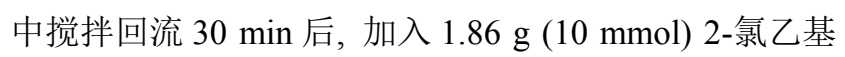
吗啉盐酸盐和催化量碘化钾, 继续回流摚拌 $9 \mathrm{~h}, \mathrm{TLC}$ 监 测至反应完全. 反应液冷却至室温, 抽滤除去碳酸钾, 浓缩滤液, 残留物加 $150 \mathrm{~mL}$ 二氯甲烷溶解, 并用水洗 $(50 \mathrm{~mL} \times 3)$. 有机相用无水硫酸镁干燥. 抽滤并浓缩滤 液, 粗产品经硅胶 (200 300 目)柱色谱[ $V$ (石油醚)： $V$ (乙酸乙酯 $)=4: 1$ ] 分离, 得到 $1.9 \mathrm{~g} \mathrm{O}$-烷基化产物 21, 同时还得到了 $\mathrm{N}-$ 烷基化产物 21-1 及其双取代产物 21-2 和 21-3.

3-[4-(2-吗啉乙氧基)喹唑啉-2-基]苯酚(21): 白色固 体, 产率 54.1\%. m.p. 125.5 126.7 ${ }^{\circ} \mathrm{C} ;{ }^{1} \mathrm{H}$ NMR (600 $\left.\mathrm{MHz}, \mathrm{CDCl}_{3}\right) \delta: 8.03(\mathrm{~d}, J=7.8 \mathrm{~Hz}, 1 \mathrm{H}, \mathrm{Ar}-\mathrm{H}), 7.81(\mathrm{~d}$, $J=7.8 \mathrm{~Hz}, 1 \mathrm{H}, \mathrm{Ar}-\mathrm{H}), 7.79 \sim 7.77(\mathrm{~m}, 2 \mathrm{H}, \mathrm{Ar}-\mathrm{H}), 7.67(\mathrm{t}$, $J=7.8 \mathrm{~Hz}, 1 \mathrm{H}, \mathrm{Ar}-\mathrm{H}), 7.05$ (t, $J=7.8 \mathrm{~Hz}, 1 \mathrm{H}, \mathrm{Ar}-\mathrm{H}), 6.73$ (dd, $J=7.8 \mathrm{~Hz}, 1.8 \mathrm{~Hz}, 1 \mathrm{H}, \mathrm{Ar}-\mathrm{H}), 4.84(\mathrm{t}, J=5.4 \mathrm{~Hz}, 2 \mathrm{H}$, $\mathrm{CH}_{2}$ ), $3.84\left(\mathrm{t}, J=4.8 \mathrm{~Hz}, 4 \mathrm{H}, \mathrm{CH}_{2}\right), 3.06(\mathrm{t}, J=5.4 \mathrm{~Hz}, 2 \mathrm{H}$, $\mathrm{CH}_{2}$ ), 2.80 (brs, $\left.4 \mathrm{H}, \mathrm{CH}_{2}\right) ;{ }^{13} \mathrm{C}$ NMR $\left(150 \mathrm{MHz}, \mathrm{CDCl}_{3}\right) \delta$ : $166.3,159.4,156.4,151.6,139.0,133.5,129.5,127.7$, 126.4, 123.5, 120.2, 118.0, 115.1, 115.0, 66.5, 63.4, 57.5, 54.1; MS (ESI) $m / z$ : $389.3[\mathrm{M}+\mathrm{K}]^{+}$. Anal. calcd for $\mathrm{C}_{20} \mathrm{H}_{21} \mathrm{~N}_{3} \mathrm{O}_{3}$ : C 68.36, H 6.02, N 11.96; found C 68.41, H 6.08, N 12.04 .

2-(3-着基苯基)-3-(2-吗啉乙基)喹唑啉-4(3H)-酮 (21-1): 白色固体, 产率 8.5\%. m.p. 109.9 111.3 ${ }^{\circ} \mathrm{C} ;{ }^{1} \mathrm{H}$ $\operatorname{NMR}\left(600 \mathrm{MHz}, \mathrm{CDCl}_{3}\right) \delta: 8.03(\mathrm{~d}, J=7.8 \mathrm{~Hz}, 1 \mathrm{H}, \mathrm{Ar}-\mathrm{H})$, 7.77 (brs, $2 \mathrm{H}, \mathrm{Ar}-\mathrm{H}), 7.53 \sim 7.51(\mathrm{~m}, 1 \mathrm{H}, \mathrm{Ar}-\mathrm{H}), 7.23(\mathrm{t}$, $J=7.8 \mathrm{~Hz}, 1 \mathrm{H}, \mathrm{Ar}-\mathrm{H}), 6.95$ (d, J=7.2 Hz, 2H, Ar-H), 6.91 (s, 1H, Ar-H), 6.74 (d, $J=8.4 \mathrm{~Hz}, 1 \mathrm{H}, \mathrm{Ar}-\mathrm{H}), 4.17$ (t, $J=$ $7.2 \mathrm{~Hz}, 2 \mathrm{H}, \mathrm{CH}_{2}$ ), 3.57 (brs, $4 \mathrm{H}, \mathrm{CH}_{2}$ ), 2.57 (t, $J=7.2 \mathrm{~Hz}$, $2 \mathrm{H}, \mathrm{CH}_{2}$ ); 2.30 (brs, $4 \mathrm{H}, \mathrm{CH}_{2}$ ); ${ }^{13} \mathrm{C}$ NMR $(150 \mathrm{MHz}$, $\left.\mathrm{CDCl}_{3}\right) \delta: 162.1,157.2,157.0,146.6,135.3,134.9,130.1$, 127.5, 127.0, 126.7, 120.8, 119.1, 117.8, 116.0, 66.7, 56.5, 53.6, 42.6; MS (ESI) $m / z: 352.3[\mathrm{M}+\mathrm{H}]^{+}$. Anal. calcd for $\mathrm{C}_{20} \mathrm{H}_{21} \mathrm{~N}_{3} \mathrm{O}_{3}$ : C 68.36, H 6.02, N 11.96; found C 68.38, H 5.97, N 11.91 .
2-[3-(2-吗啉乙氧基)苯基]-3-(2-吗啉乙基)喹唑啉4(3H)-酮(21-2): 白色固体，产率 4.6\%. m.p. 113.3 $114.7{ }^{\circ} \mathrm{C} ;{ }^{1} \mathrm{H}$ NMR $\left(600 \mathrm{MHz}, \mathrm{CDCl}_{3}\right) \delta: 8.30(\mathrm{~d}, J=8.4$ $\mathrm{Hz}, 1 \mathrm{H}, \mathrm{Ar}-\mathrm{H}), 7.75$ (t, $J=7.2 \mathrm{~Hz}, 1 \mathrm{H}, \mathrm{Ar}-\mathrm{H}), 7.71$ (d, $J=$ $8.4 \mathrm{~Hz}, 1 \mathrm{H}, \mathrm{Ar}-\mathrm{H}), 7.50$ (t, J=7.2 Hz, 1H, Ar-H), 7.40 (t, $J=7.8 \mathrm{~Hz}, 1 \mathrm{H}, \mathrm{Ar}-\mathrm{H}), 7.11$ (t, $J=7.8 \mathrm{~Hz}, 1 \mathrm{H}, \mathrm{Ar}-\mathrm{H}), 7.08$ (s, $1 \mathrm{H}, \operatorname{Ar}-\mathrm{H}), 7.03$ (d, $J=8.4 \mathrm{~Hz}, 1 \mathrm{H}, \mathrm{Ar}-\mathrm{H}), 4.15$ (q, $J=$ $\left.6.6 \mathrm{~Hz}, 4 \mathrm{H}, \mathrm{CH}_{2}\right), 3.72\left(\mathrm{t}, J=4.8 \mathrm{~Hz}, 4 \mathrm{H}, \mathrm{CH}_{2}\right), 3.54(\mathrm{t}$, $\left.J=4.2 \mathrm{~Hz}, 4 \mathrm{H}, \mathrm{CH}_{2}\right), 2.82\left(\mathrm{t}, J=6.0 \mathrm{~Hz}, 2 \mathrm{H}, \mathrm{CH}_{2}\right), 2.58$ (brs, $4 \mathrm{H}, \mathrm{CH}_{2}$ ), 2.54 (t, $J=6.6 \mathrm{~Hz}, 2 \mathrm{H}, \mathrm{CH}_{2}$ ), 2.2.25 (brs, $\left.4 \mathrm{H}, \mathrm{CH}_{2}\right) ;{ }^{13} \mathrm{C}$ NMR $\left(150 \mathrm{MHz}, \mathrm{CDCl}_{3}\right) \delta: 162.3,159.0$, $155.9,147.2,136.7,134.5,130.1,127.6,127.2,126.8$, $120.9,120.6,116.1,114.8,67.0,66.9,66.2,57.6,56.8$, 54.2, 53.8, 42.8; MS (ESI) $m / z: 488.2[\mathrm{M}+\mathrm{Na}]^{+}$. Anal. calcd for $\mathrm{C}_{26} \mathrm{H}_{32} \mathrm{~N}_{4} \mathrm{O}_{4}$ : C 67.22, H 6.94, N 12.06; found $\mathrm{C}$ 67.26, H 6.98, N 12.17 .

4-\{2-\{\{2-[3-(2-吗啉乙氧基)苯基]喹唑啉-4-基\}氧 基 \} 乙基\} 吗啉 (21-3): 白色固体, 产率 5.5\%. m.p. 129.7 131.1 ${ }^{\circ} \mathrm{C} ;{ }^{1} \mathrm{H}$ NMR (600 MHz, $\left.\mathrm{CDCl}_{3}\right) \delta: 8.17(\mathrm{~d}$, $J=7.8 \mathrm{~Hz}, 1 \mathrm{H}, \mathrm{Ar}-\mathrm{H}), 8.14$ (d, $J=7.2 \mathrm{~Hz}, 2 \mathrm{H}, \mathrm{Ar}-\mathrm{H}), 7.98$ (d, $J=8.4 \mathrm{~Hz}, 1 \mathrm{H}, \mathrm{Ar}-\mathrm{H}), 7.82$ (t, $J=7.8 \mathrm{~Hz}, 1 \mathrm{H}, \mathrm{Ar}-\mathrm{H}$ ), $7.51(\mathrm{t}, J=7.2 \mathrm{~Hz}, 1 \mathrm{H}, \mathrm{Ar}-\mathrm{H}), 7.40(\mathrm{t}, J=8.4 \mathrm{~Hz}, 1 \mathrm{H}$, Ar-H), 7.04 (dd, $J=8.4,2.4 \mathrm{~Hz}, 1 \mathrm{H}, \mathrm{Ar}-\mathrm{H}), 4.86$ (t, $J=7.2$ $\left.\mathrm{Hz}, 2 \mathrm{H}, \mathrm{CH}_{2}\right), 4.26\left(\mathrm{t}, J=5.4 \mathrm{~Hz}, 2 \mathrm{H}, \mathrm{CH}_{2}\right), 3.76$ (t, $J=4.8$ $\left.\mathrm{Hz}, 4 \mathrm{H}, \mathrm{CH}_{2}\right), 3.74$ (t, $\left.J=4.2 \mathrm{~Hz}, 4 \mathrm{H}, \mathrm{CH}_{2}\right), 2.97$ (t, $J=5.4$ $\mathrm{Hz}, 2 \mathrm{H}, \mathrm{CH}_{2}$ ), 2.87 (t, $J=5.4 \mathrm{~Hz}, 2 \mathrm{H}, \mathrm{CH}_{2}$ ), 2.66 (brs, $4 \mathrm{H}$, $\mathrm{CH}_{2}$ ), 2.63 (brs, $\left.4 \mathrm{H}, \mathrm{CH}_{2}\right) ;{ }^{13} \mathrm{C} \mathrm{NMR}\left(150 \mathrm{MHz}, \mathrm{CDCl}_{3}\right) \delta$ : $166.6,159.8,159.1,152.0,139.7,133.7,129.5,128.1$, 126.6, 123.6, 121.4, 117.2, 115.4, 114.3, 67.1, 67.0, 66.0, 64.6, 57.9, 57.3, 54.2, 54.2; MS (ESI) $m / z: 465.4[\mathrm{M}+$ $\mathrm{H}]^{+}$. Anal. calcd for $\mathrm{C}_{26} \mathrm{H}_{32} \mathrm{~N}_{4} \mathrm{O}_{4}$ : C 67.22, H 6.94, N 12.06; found C 67.35, H 6.88, N 12.10 .

\section{7 化合物 22 的合成}

$100 \mathrm{~mL}$ 圆底烧瓶中加入 $523 \mathrm{mg}(1.5 \mathrm{mmol})$ 化合物 21, $554 \mathrm{mg}(2.3 \mathrm{mmol})$ 邻碘苯甲酸, $3.7 \mathrm{mg}(4.0 \mathrm{mmol})$ DCC, $18 \mathrm{mg}$ (0.2 mmol) DMAP. 于 $30 \mathrm{~mL}$ 新处理的无水 四氢呋喃中室温搅拌, TLC 监测至反应完全. 抽滤并浓 缩滤液, 粗产品用 $[V$ (石油醚 $): V($ 乙酸乙酯 $)=1: 1)$ 重 结晶得到 $824 \mathrm{mg}$ 2-碘苯甲酸 3-[4-(2-吗啉乙氧基)喹唑 啉-2-基]苯酯(22), 白色固体, 产率 95.3\%. m.p. 48.1 $49.7{ }^{\circ} \mathrm{C} ;{ }^{1} \mathrm{H}$ NMR $\left(600 \mathrm{MHz}, \mathrm{CDCl}_{3}\right) \delta: 8.52(\mathrm{~d}, J=7.8$ $\mathrm{Hz}, 1 \mathrm{H}, \mathrm{Ar}-\mathrm{H}), 8.46$ (s, 1H, Ar-H), 8.14 (d, $J=7.8 \mathrm{~Hz}, 1 \mathrm{H}$, Ar-H), 8.11 (dd, $J=6,1.2 \mathrm{~Hz}, 1 \mathrm{H}, \mathrm{Ar}-\mathrm{H}), 8.09$ (d, $J=7.8$ $\mathrm{Hz}, 1 \mathrm{H}, \mathrm{Ar}-\mathrm{H}), 7.98$ (d, $J=7.2 \mathrm{~Hz}, 1 \mathrm{H}, \mathrm{Ar}-\mathrm{H}), 7.82$ (t, $J=$ 
$7.5 \mathrm{~Hz}, 1 \mathrm{H}, \mathrm{Ar}-\mathrm{H}), 7.57$ (t, $J=7.8 \mathrm{~Hz}, 1 \mathrm{H}, \mathrm{Ar}-\mathrm{H}), 7.52$ (t, $J=7.8 \mathrm{~Hz}, 1 \mathrm{H}, \mathrm{Ar}-\mathrm{H}), 7.57$ (t, $J=7.2 \mathrm{~Hz}, 1 \mathrm{H}, \mathrm{Ar}-\mathrm{H}), 8.11$ (dd, $J=6.0,1.8 \mathrm{~Hz}, 1 \mathrm{H}$, Ar-H), 7.23 (t, $J=7.2 \mathrm{~Hz}, 1 \mathrm{H}$, Ar-H), 4.85 (t, $J=6 \mathrm{~Hz}, 2 \mathrm{H}, \mathrm{CH}_{2}$ ), 3.73 (t, $J=4.5 \mathrm{~Hz}, 2 \mathrm{H}$, $\mathrm{CH}_{2}$ ), 2.96 (t, $J=6 \mathrm{~Hz}, 2 \mathrm{H}, \mathrm{CH}_{2}$ ), 2.65 (brs, $4 \mathrm{H}, \mathrm{CH}_{2}$ ); ${ }^{13} \mathrm{C}$ NMR $\left(150 \mathrm{MHz}, \mathrm{CDCl}_{3}\right) \delta: 166.7,164.9,158.9,151.9$, $151.1,141.8,140.0,134.2,133.7,133.4,131.7,129.5$, $128.2,128.1,126.8,126.2,123.8,123.6,121.6,115.5$, 94.9, 67.1, 64.6, 57.3, 54.2; MS (ESI) $m / z: 620.7[\mathrm{M}+$ $\mathrm{K}]^{+}$.

\section{8 化合物 $4 a$ 的合成}

$50 \mathrm{~mL}$ 圆底烧瓶中加入 $228 \mathrm{mg}(0.4 \mathrm{mmol}) \mathbf{2 2}, 21$ $\mathrm{mg}(0.02 \mathrm{mmol})$ 三苯基膦, $49 \mathrm{mg}(0.6 \mathrm{mmol})$ 醋酸钠, 9 $\mathrm{mg}(0.04 \mathrm{mmol})$ 醋酸钯, 于 $10 \mathrm{~mL}$ 新处理的 $\mathrm{DMF}$ 中 $120{ }^{\circ} \mathrm{C}$ 油浴加热. TLC 监测至反应结束后, 减压蒸出大 部分溶剂, 残留物加 $30 \mathrm{~mL}$ 二氯甲烷溶解, 抽滤除去不 溶物. 滤液用 $10 \mathrm{~mL}$ 水洗 3 次, 有机相用无水硫酸镁干 燥. 抽滤并浓缩滤液, 粗产品经硅胶 (200 300 目)柱色 谱 $[V($ 二氯甲烷 $): V$ (甲醇 $)=40: 1$ )分离, 得到 $50 \mathrm{mg}$ 3-[4-(2-吗啉乙氧基)喹唑啉-2-基]- $6 H$-苯并 $[c$ ]苯并二氢 吡喃-6-酮(4a), 白色固体, 产率 28.4\%. m.p. 126.5 $127.7{ }^{\circ} \mathrm{C} ;{ }^{1} \mathrm{H}$ NMR $\left(600 \mathrm{MHz}, \mathrm{CDCl}_{3}\right) \delta: 8.57$ (d, $J=4.8$ $\mathrm{Hz}, 2 \mathrm{H}, \mathrm{Ar}-\mathrm{H}$ ), 8.43 (d, $J=7.2 \mathrm{~Hz}, 1 \mathrm{H}, \mathrm{Ar}-\mathrm{H}), 8.20$ (d, $J=$ $7.8 \mathrm{~Hz}, 1 \mathrm{H}, \mathrm{Ar}-\mathrm{H}), 8.16$ (d, J=7.8 Hz, 2H, Ar-H), 8.01 (d, $J=8.4 \mathrm{~Hz}, 1 \mathrm{H}, \mathrm{Ar}-\mathrm{H}), 7.87 \sim 7.84(\mathrm{~m}, 2 \mathrm{H}, \mathrm{Ar}-\mathrm{H}), 7.62(\mathrm{t}$, $J=7.2 \mathrm{~Hz}, 1 \mathrm{H}, \mathrm{Ar}-\mathrm{H}), 7.56$ (t, $J=7.2 \mathrm{~Hz}, 2 \mathrm{H}, \mathrm{Ar}-\mathrm{H}), 4.91$ (t, $\left.J=6.0 \mathrm{~Hz}, 2 \mathrm{H}, \mathrm{CH}_{2}\right), 3.79\left(\mathrm{t}, J=4.2 \mathrm{~Hz}, 4 \mathrm{H}, \mathrm{CH}_{2}\right), 3.04$ (t, $J=6.0 \mathrm{~Hz}, 2 \mathrm{H}, \mathrm{CH}_{2}$ ), 2.72 (brs, $4 \mathrm{H}, \mathrm{CH}_{2}$ ); ${ }^{13} \mathrm{C}$ NMR $\left(150 \mathrm{MHz}, \mathrm{CDCl}_{3}\right) \delta: 166.8,161.4,158.4,151.9,151.6$, $140.6,135.0,134.7,133.9,130.8,129.3,128.3,127.1$, $124.4,123.7,122.9,122.3,121.6,119.8,117.9,115.6$, 67.0, 64.7, 67.3, 54.2; MS (ESI) $m / z: 477.1[\mathrm{M}+\mathrm{Na}]^{+}$. Anal. calcd for $\mathrm{C}_{27} \mathrm{H}_{23} \mathrm{~N}_{3} \mathrm{O}_{4}$ : C 71.51, H 5.11, N 9.27; found $\mathrm{C} 71.62, \mathrm{H} 5.08, \mathrm{~N} 9.32$.

\section{9 抗肿瘤细胞毒性测试}

抗肿瘤细胞毒性采用改进的 Mosmann's 噻唑蓝 (MTT) 染色法测定 ${ }^{[16]}$. 具体操作如下: 于 96 孔板接种细 胞悬液, $90 \mu \mathrm{L}$ 孔, 细胞密度 $2 \times 10^{4}$ 个 $/ \mathrm{mL}, 37{ }^{\circ} \mathrm{C}, 5 \%$ $\mathrm{CO}_{2}$ 浓度的培养箱中过夜, 加 $10 \mu \mathrm{L} /$ 孔相应浓度药物 $(100 \mu \mathrm{mol} / \mathrm{L})$, 继续培 $44 \mathrm{~h}$, 加 MTT $10 \mu \mathrm{L}$ 孔培养 $4 \mathrm{~h}$, 从培养箱中取出, 倒板, 加 DMSO $100 \mu \mathrm{L}$ 孔溶解 MTT, 测量 $570 \mathrm{~nm}$ 波长下肿瘤细胞 $\mathrm{OD}$ 值, 代入公式: $\left(\mathrm{OD}_{\text {control }}-\mathrm{OD}_{\text {treated }}\right) / \mathrm{OD}_{\text {control }} \times 100 \%$ 计算抑制率，顺铂 为阳性对照.

\subsection{0 抗菌活性测试方法}

细菌测试方法: 各吸取 $10^{-6}$ 或 $10^{-7}$ 梯度菌悬液 70 $\mu \mathrm{L}$, 分别加入到药物浓度为 $50 \mu \mathrm{g} / \mathrm{mL}$ 的药物平板和不 加药的营养琼脂(NA)培养基平板(对照)上，用灭菌三角 刮涂布均匀, 做好标识, 置于 $37{ }^{\circ} \mathrm{C}$ 恒温培养箱中培养 24 h. $24 \mathrm{~h}$ 后, 进行菌落计数, 每组设置 3 次平行试验.

抑菌率 $=($ 对照组菌落数 - 药物平板菌落数 $) /$

对照组菌落数 $\times 100 \%$

真菌测试方法: 将供试化合物用质量分数为 $5 \%$ 的 二甲基亚砜(DMSO)水溶液配制成质量浓度分别为 50 $\mu \mathrm{g} / \mathrm{mL}$ 马铃薯葡萄糖琼脂(PDA)培养基, 测试其对 4 种 供试真菌病原菌的抑菌活性. 在已培养好供试菌株的平 皿中取直径 $8 \mathrm{~mm}$ 的菌饼, 置于含药平板内, 每血 3 块. 以不加药剂者为空白对照. 于温度为 $(25 \pm 1){ }^{\circ} \mathrm{C}$ 培养箱 内培养 $5 \mathrm{~d}$, 采用十字交叉法测量菌落直径, 计算菌丝 生长抑制率. 计算公式如下:

\section{菌落增长直径 $=$ 菌落直径一菌饼直径}

抑制率 $=($ 对照菌落增长直径 - 试验菌落增长直 径)/(对照菌落增长直径) $\times 100 \%$

\section{辅助材料(Supporting Information) 化合物 $3 \mathrm{a} \sim 3 \mathrm{e} 、$} $3 b^{\prime} \sim 3 e^{\prime} 、 4 a$ 和 $21 、 21-1 、 21-2 、 21-3$ 的核磁共振氢谱 和碳谱. 这些材料可以免费从本刊网站 (http://siocjournal.cn/)上下载.

\section{References}

[1] Khan, I.; Zaib, S.; Batool, S.; Abbas, N.; Ashraf, Z.; Iqbal, J.; Saeed, A. Bioorg. Med. Chem. 2016, 24, 2361.

[2] Khan, I.; Ibrar, A.; Abbas, N.; Saeed, A. Eur. J. Med. Chem. 2014, 76, 193.

[3] Yan, B. R.; Lv, X. Y.; Du, H.; Bao, X. P. Chin. J. Org. Chem. 2016, 36, 207 (in Chinese). (问柏任，吕新阳，杜欢，鲍小平，有机化学, 2016, 36, 207.)

[4] Khan, I.; Ibrar, A.; Ahmed, W.; Saeed, A. Eur. J. Med. Chem. 2015, $90,124$.

[5] Ou, J. J.; Liu, K. C.; Wang, Y.; Zhang, H.; Liu, R. Q.; Li, Q. B.; Wang, Q. M.; Li, Y. Q.; Rui, C. H.; Liu, S. Z. Chin. J. Org. Chem. 2014, 34, 526 (in Chinese). (欧俊军，刘克昌，王毅，张浩，刘瑞全，李奇博，汪清民，李永 强, 芮昌辉, 刘尚钟, 有机化学, 2014, 34, 526.)

[6] Xu, W.; Jin, Y. B.; Liu, H. X.; Jiang, Y. Y.; Fu, H. Org. Lett. 2011, $13,1274$.

[7] Liu, C. E.; Yu, Q. Y.; Tang, J. H.; Li, J. R. Chin. J. Org. Chem. 2012, 32, 532 (in Chinese). (刘长娥, 于琪瑶, 唐健红, 李加荣, 有机化学, 2012, 32, 532.)

[8] Alam, A.; Tsuboi, S. Tetrahedron 2007, 63, 10454.

[9] Bringmann, G.; Menche, D.; Mulhlbacher, J.; Reichert, M.; Saito, N.; Pfeiffer, S. S.; Lipshutz, B. H. Org. Lett. 2002, 4, 2833.

[10] Wang, Z. T.; Zhang, Y. S.; Wang, S. C.; Xia, D. H. Chin. J. Org. Chem. 2007, 27, 143 (in Chinese). (王宗廷, 张云山, 王书超, 夏道宏, 有机化学, 2007, 27, 143.)

[11] Xu, S.; Xu, S. P.; Li, L. M. Acta Pharm. Sin. 2002, 37, 113 (in Chi- 
nese).

(徐嵩, 徐世平, 李兰敏, 药学学报, 2002, 37, 113.)

[12] Kim, N. Y.; Cheon, C.-H. Tetrahedron Lett. 2014, 55, 2340.

[13] Wang, Z. Z.; Tang, Y. Tetrahedron 2016, 72, 1330.
[14] Chen, G. S.; Kalchar, S.; Kuo, C.-W.; Chang, C.-S.; Usifoh, C. O.; Chern, J.-W. J. Org. Chem. 2003, 68, 2502.

[15] Hori, M.; Ohtaka, H. Chem. Pharm. Bull. 1993, 41, 1114.

[16] Mosmann, T. J. Immunol. Methods 1983, 65, 55.

(Qin, X.) 